\title{
DUOX1 mediates persistent epithelial EGFR activation, mucous cell metaplasia, and airway remodeling during allergic asthma
}

Aida Habibovic, ${ }^{1}$ Milena Hristova, ${ }^{1}$ David E. Heppner, ${ }^{1}$ Karamatullah Danyal, ${ }^{1}$ Jennifer L. Ather, ${ }^{2}$ Yvonne M.W. Janssen-Heininger, ${ }^{1}$ Charles C. Irvin, ${ }^{2}$ Matthew E. Poynter, ${ }^{2}$ Lennart K. Lundblad, ${ }^{2}$ Anne E. Dixon, ${ }^{2}$ Miklos Geiszt, ${ }^{3}$ and Albert van der Vliet ${ }^{1}$

'Departments of Pathology and Laboratory Medicine and 'Department of Medicine, Vermont Lung Center, Robert Larner, M.D. College of Medicine, University of Vermont, Burlington, Vermont, USA. 'Department of Physiology and "Lendület" Peroxidase Enzyme Research Group, Semmelweis University, Budapest, Hungary.

Chronic inflammation with mucous metaplasia and airway remodeling are hallmarks of allergic asthma, and these outcomes have been associated with enhanced expression and activation of EGFR signaling. Here, we demonstrate enhanced expression of EGFR ligands such as amphiregulin as well as constitutive EGFR activation in cultured nasal epithelial cells from asthmatic subjects compared with nonasthmatic controls and in lung tissues of mice during house dust mite-induced (HDM-induced) allergic inflammation. EGFR activation was associated with cysteine oxidation within EGFR and the nonreceptor tyrosine kinase Src, and both amphiregulin production and oxidative EGFR activation were diminished by pharmacologic or genetic inhibition of the epithelial NADPH oxidase dual oxidase 1 (DUOX1). DUOX1 deficiency also attenuated several EGFR-dependent features of HDM-induced allergic airway inflammation, including neutrophilic inflammation, type 2 cytokine production (IL-33, IL-13), mucous metaplasia, subepithelial fibrosis, and central airway resistance. Moreover, targeted inhibition of airway DUOX1 in mice with previously established HDM-induced allergic inflammation, by intratracheal administration of DUOX1-targeted siRNA or pharmacological NADPH oxidase inhibitors, reversed most of these outcomes. Our findings indicate an important function for DUOX 1 in allergic inflammation related to persistent EGFR activation and suggest that DUOX1 targeting may represent an attractive strategy in asthma management.

Authorship note: A. Habibovic and M. Hristova contributed equally to this work.

Conflict of interest: The authors have declared that no conflict of interest exists.

Submitted: June 6, 2016 Accepted: September 30, 2016 Published: November 3, 2016

Reference information: JCI Insight. 2016;1(18):e88811. doi:10.1172/jici.insight.88811

\section{Introduction}

Allergic airway diseases are rapidly increasing worldwide and form a significant health burden. While many forms of asthma are successfully managed with current treatments with corticosteroids and/or bronchodilators, many asthmatics still suffer from poor management due to steroid resistance or life-threatening exacerbations $(1,2)$. Asthma is commonly viewed as a disease dominated by enhanced adaptive immune responses leading to eosinophilic inflammation, but recent evidence indicates that innate immune responses originating from the respiratory epithelium contribute importantly to enhanced inflammation as well as mucous metaplasia and airway remodeling, which are important hallmarks of allergic asthma $(3,4)$. Moreover, genetic or epigenetic alterations contribute to an altered respiratory epithelium, which is hyperreactive to allergen challenges and thereby mediates exaggerated responses to allergens during exacerbations $(3,5)$.

One well-recognized feature of epithelial alterations in the asthmatic airway is the increased expression of the EGFR and several of its ligands, leading to increased and corticosteroid-insensitive EGFR activation (6-10). Epithelial EGFR activation contributes to several important features of asthma, including the activation of a chronic wound response within the airways characterized by airway remodeling, mucous metaplasia, and neutrophilic inflammation (11-13). Moreover, our recent findings indicate that epithelial EGFR activation also mediates allergen-induced epithelial secretion of the alarmin IL-33 and subsequent activation of type 2 inflammatory responses (14). Accordingly, several studies in animal models have demonstrated that inhibition of epithelial EGFR can prevent or inhibit many of these cardinal features; 
in particular, it affects neutrophilic inflammation, mucous metaplasia, subepithelial fibrosis, and airway resistance (15-17). However, these findings have not been translated into clinical use of available selective EGFR tyrosine kinase inhibitors in treatment of asthma, as EGFR tyrosine kinase inhibitors are associated with adverse effects, such as enhanced skin rash and inflammation (18). In addition, while EGFR tyrosine kinase inhibitors are used successfully in the treatment of lung cancers, they commonly induce acquired resistance due to the promotion of secondary EGFR mutations (19), thus significantly limiting their use in less life-threatening chronic diseases. A recent clinical trial using the EGFR inhibitor BIBW 2948 in patients with COPD indicated efficacy with respect to inhibiting EGFR activation and a tendency toward reducing mucous metaplasia but was terminated due to adverse effects such as reversible liver enzyme elevation and decreased forced expiratory volume in 1 second (FEV1) in some patients (20). Therefore, alternative strategies to more selectively prevent or suppress airway EGFR activation in the context of chronic or severe asthma, while avoiding these adverse outcomes, would be desirable.

In spite of the strong evidence implicating chronic epithelial EGFR expression and activation in the pathophysiology of asthma, the proximal mechanisms leading to such persistent EGFR activation are poorly understood. Our recent studies as well as those by others have revealed an oxidative mechanism of airway EGFR activation in response to injury or to challenge with common airborne allergens that involves initial activation of the epithelial NADPH oxidase dual oxidase 1 (DUOX1) (14, 21, 22). DUOX1-mediated EGFR activation involves a cascade of events, including activation of the redox-sensitive nonreceptor tyrosine kinase Src and production of soluble EGFR ligands $(23,24)$. In addition, DUOX1 also contributes to enhanced EGFR tyrosine kinase activity by promoting cysteine oxidation within EGFR itself (14, 25). The relevance of these findings for asthma is supported by recent findings that DUOX1 expression is enhanced in nasal epithelial cells (NECs) from subjects with allergic asthma and rhinosinusitis $(14,26)$. Based on these considerations, we hypothesized that enhanced epithelial DUOX1 activation may contribute to persistent EGFR activation, activation of type 2 immune responses, and related pathological events during allergic asthma, such as mucous metaplasia, airway remodeling, and hyperresponsiveness, and that selective targeting of DUOX1 may attenuate these outcomes. The present studies indeed indicate that DUOX1 critically contributes to sustained EGFR activation during allergic asthma through direct epithelial oxidative mechanisms and indirect mechanisms involving activation of type 2 innate lymphoid cells (ILC2s) and production of the EGFR ligand amphiregulin (AREG). Moreover, DUOX1 was found to contribute to cardinal features of allergic inflammation and remodeling in a mouse model of house dust mite-induced (HDM-induced) allergic asthma, and genetic and pharmacological targeting of DUOX1 was able to reverse these outcomes.

\section{Results}

EGFR phosphorylation and cysteine oxidation are enhanced in NECs from asthmatic subjects. We recently collected NECs from a cohort of subjects with allergic asthma and nonasthmatic controls, which indicated enhanced DUOX1 expression in asthmatic epithelia (14). Secondary analysis of protein extracts or cDNAs from these NECs indicated a consistent presence of activated (phosphorylated) EGFR as well as Src, a critical mediator of EGFR activation (23), in NECs from asthmatic subjects compared with nonasthmatic controls (Figure 1A), in addition to dramatically increased mRNA expression of several EGFR ligands, with 2- to 6-fold increases in EGF and TGFA mRNA and 4- to 90-fold increases in $A R E G$ in NECs from subjects with asthma (Figure 1B). Moreover, the extent of EGFR phosphorylation at tyrosine 1068 and AREG mRNA expression in NECs from subjects with asthma in both cases inversely correlated with reduced lung function, as measured as percentage of FEV1 (Figure 1C). Because NECs from asthmatic subjects contain increased levels of DUOX1 (14), we postulated that increased EGFR and Src activation in asthmatic epithelia was associated with increased cysteine oxidation of these kinases. To address this, we cultured NECs from healthy and asthmatic subjects to confluence and harvested cell lysates after overnight culture in EGF-depleted culture medium in the presence of DCP-Bio1, a dimedone-based derivatizing agent that reacts with protein sulfenic acids (-SOH, the primary cysteine oxidation product) to add a biotin tag. Whereas no detectable phosphorylation of EGFR or Src was observed in NECs from nonasthmatic controls in this case, marked increases in EGFR and Src phosphorylation were detected in asthmatic NECs (Figure 1D), which most likely coincided with increased EGFR ligand expression (Figure 1B). Moreover, these increases in EGFR/Src phosphorylation coincided with marked increases in cysteine oxidation within these proteins, as determined by Western blot 
A

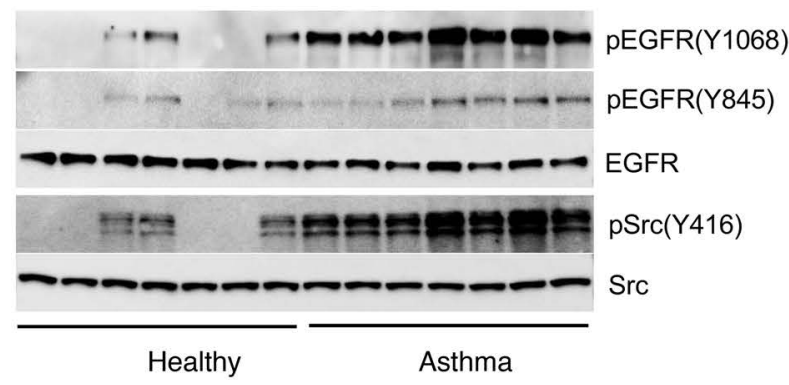

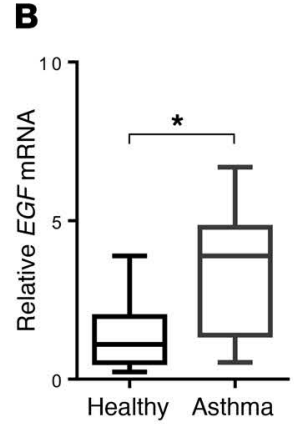
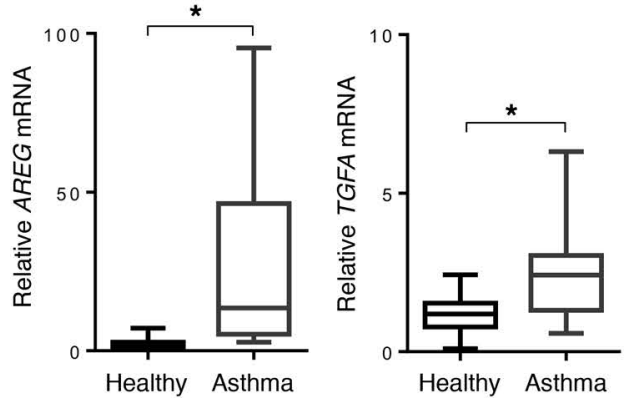

C

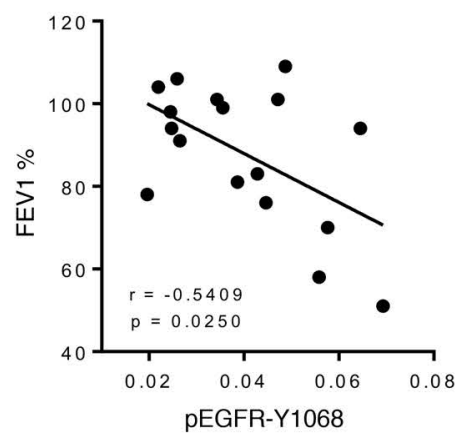

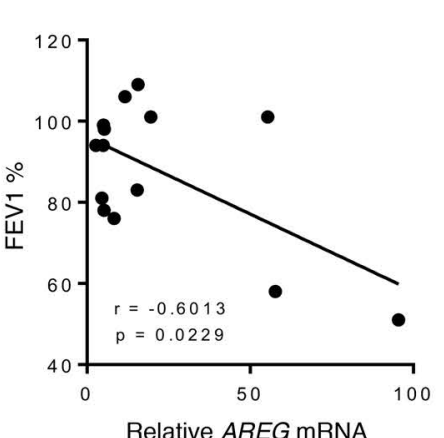

D

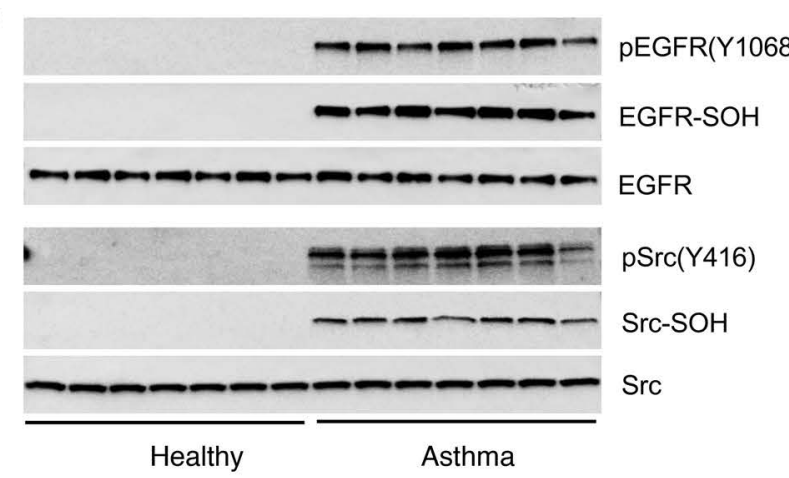

E
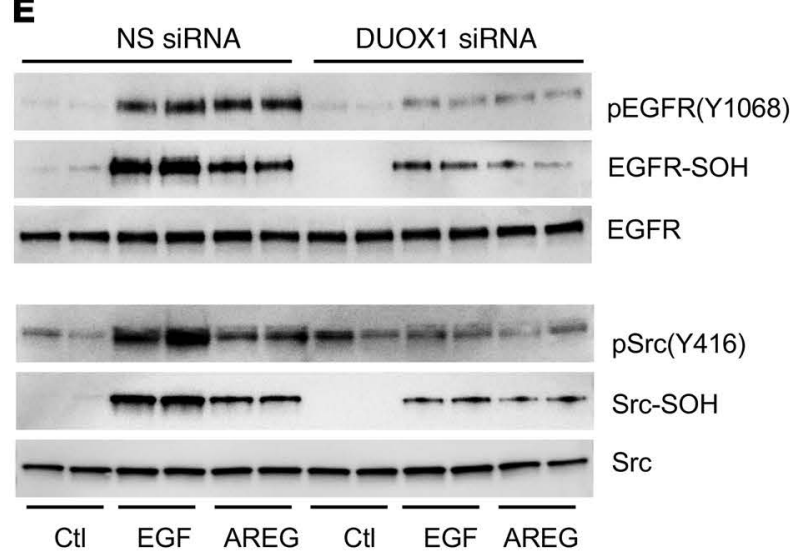

F
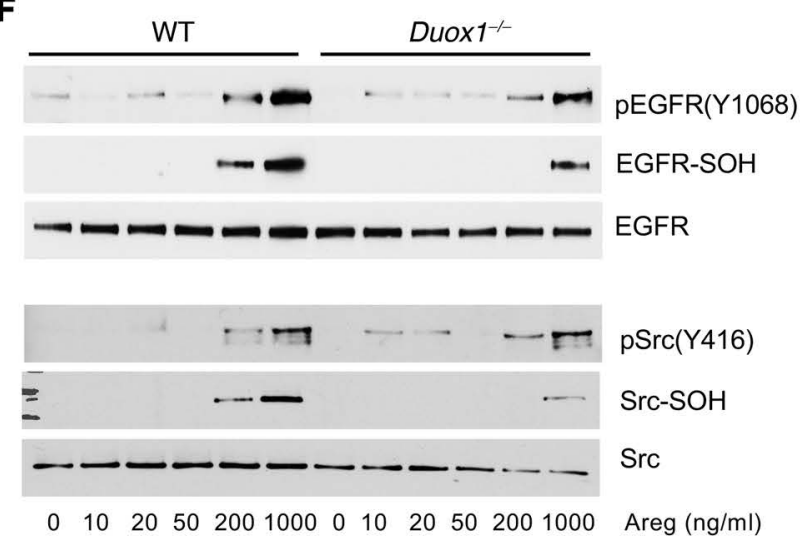

Figure 1. EGFR activation and oxidation are enhanced in nasal epithelial cells from asthmatic subjects. (A) Western blot of phosphorylation status of EGFR and Src in primary cultures of NECs from 7 healthy and 7 asthmatic subjects. (B) RT-PCR analysis of EGFR ligands EGF, AREG, and TGF- $\alpha$ (TCFA) in NECs from healthy $(n=17)$ and asthmatic $(n=13)$ subjects; box, 25th-75th quartiles; whiskers, minimums and maximums. ${ }^{*} P<0.01$ by 2-tailed unpaired $t$ test. (C) Inverse correlation between percentage of FEV1 and EGFR phosphorylation status (determined by densitometry of pEGFR/EGFR ratio) as well as relative AREG mRNA expression in NECs from asthmatic subjects. (D) Western blot analysis of EGFR and Src phosphorylation in association with cysteine oxidation in cultured NECs from 7 healthy and 7 asthmatic subjects, determined with phospho-specific antibodies and analysis of avidin-purified proteins after DCP-Bio1 derivatization of oxidized cysteines (-SOH). (E and F) Analysis of EGF- or AREG-induced activation and cysteine oxidation in HBE1 cells transfected with either NS siRNA or DUOX1 siRNA (E) or mTECs from wild-type or Duox1 ${ }^{-1-}$ mice (F). Representative blots of at least 2 independent experiments are shown.

analysis of avidin-purified proteins from DCP-Bio1-derivatized NEC lysates for EGFR or Src (Figure 1D). These findings indicate the presence of persistent EGFR activation in asthmatic epithelia, which is likely sustained by autocrine or paracrine EGFR ligand production and enhanced oxidative mechanisms that promote EGFR activation. To address the role of DUOX1 in such ligand-induced EGFR activation and cysteine oxidation, we used the human bronchial epithelial cell line HBE1 and evaluated tyrosine phosphorylation and cysteine oxidation of EGFR and Src after stimulation with EGF or AREG. In both cases, ligand-induced stimulation of phosphorylation and oxidation of EGFR and Src were attenuated 
A HDM extract/PBS

$(50 \mu \mathrm{g}$, i.n.)

$\downarrow \downarrow \downarrow \downarrow \downarrow \quad \downarrow \downarrow \downarrow \downarrow \downarrow \quad \downarrow \downarrow \downarrow \downarrow \downarrow$

Day 0

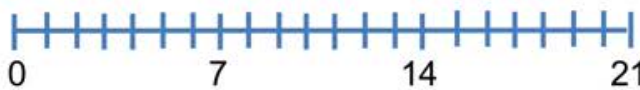

C

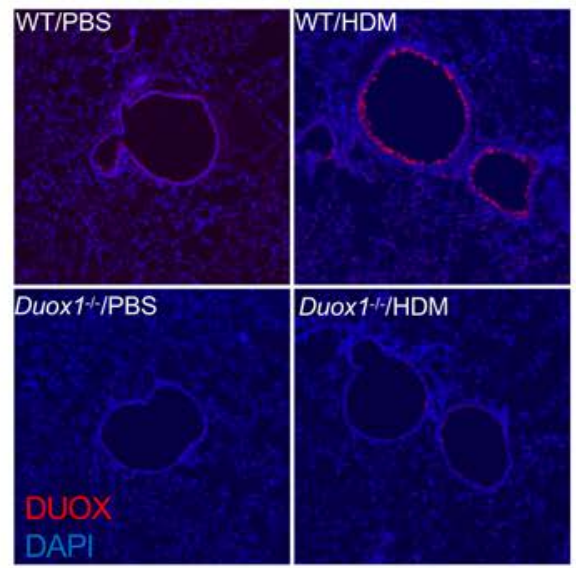

D

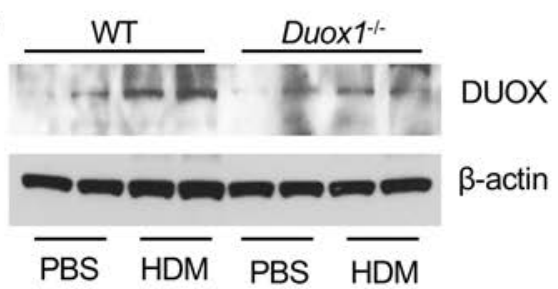

$\downarrow$ harvest
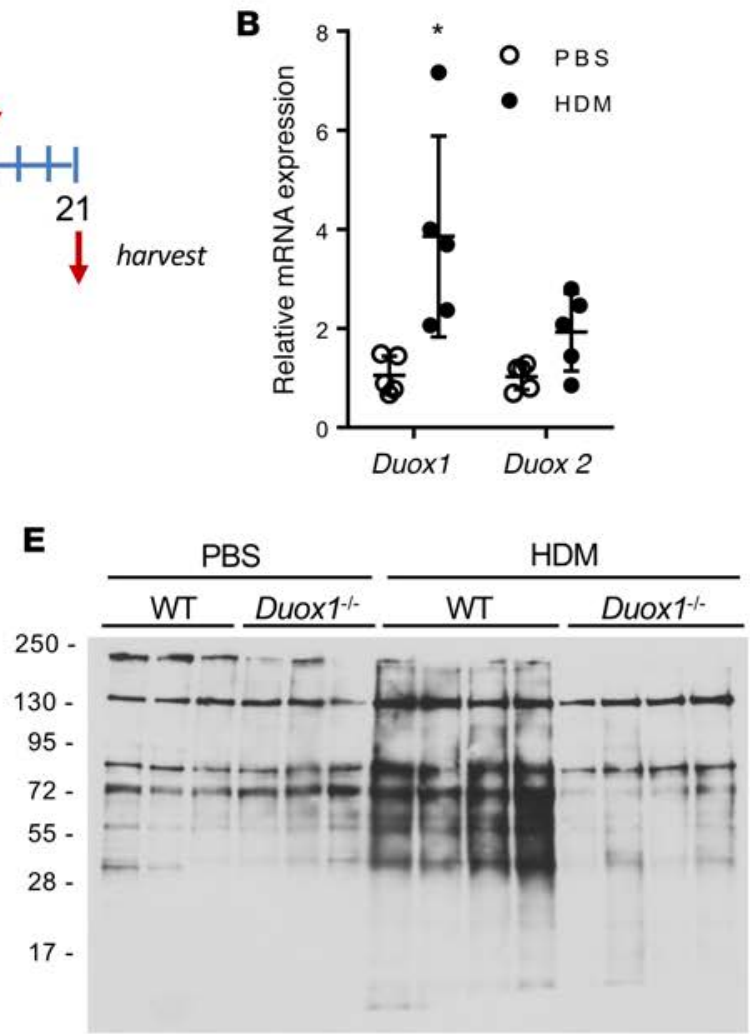

Label: DCP-Bio1; Blot: Streptavidin

$\mathbf{F}$

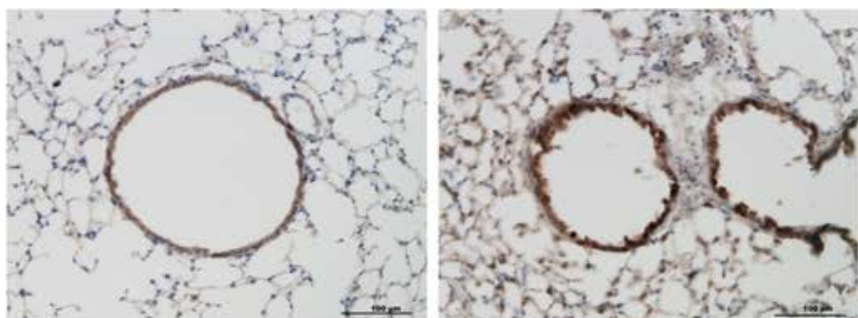

WT/PBS

G

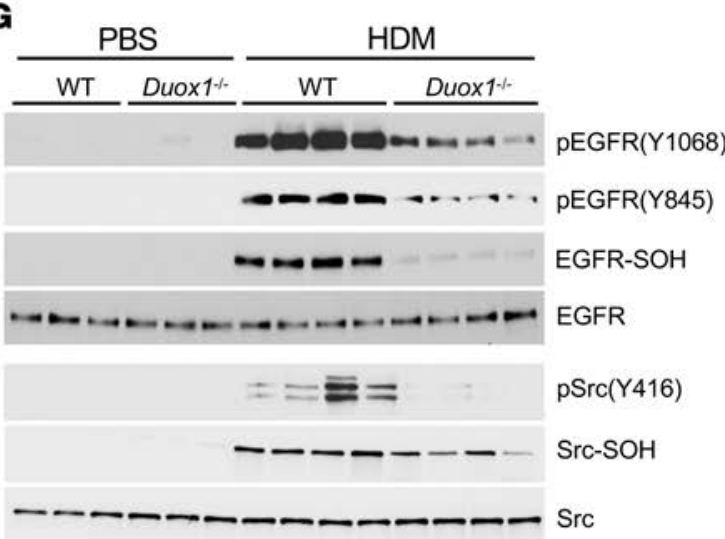

H
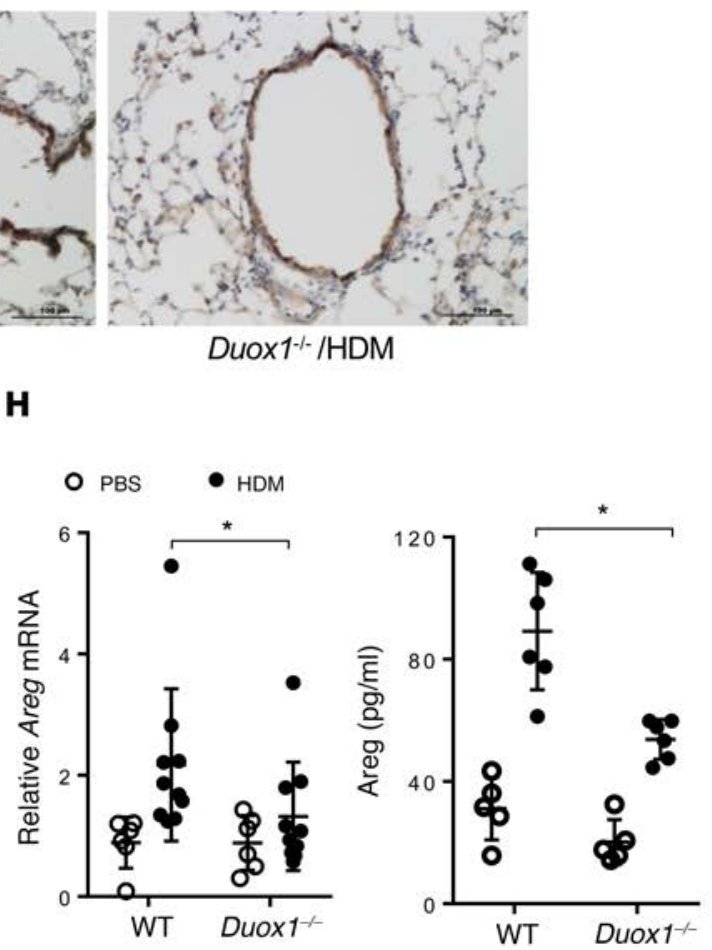
Figure 2. DUOX1 contributes to oxidative EGFR activation in house dust mite-induced allergic asthma in mice. (A) Experimental design of house dust mite-induced (HDM-induced) allergic inflammation. (B) Analysis of DUOX1 and DUOX2 mRNA in lung tissues. (C and D) Analysis of DUOX1 protein expression in lung tissues using immunofluorescence (C; nuclei counterstained in blue; original magnification $\times 10$ ) or Western blot analysis (D). (E) Analysis of protein cysteine sulfenylation by DCP-Bio1 labeling of lung tissue homogenates and streptavidin blotting. (F and $\mathbf{G})$ Analysis of EGFR activation by IHC for phosphorylated EGFR (Y1068) (F) and Western blot analysis of lung tissue homogenates for tyrosine phosphorylation and cysteine oxidation (-S-OH, using DCP-Bio1 labeling) within EGFR or Src (G). Scale bars: $100 \mu \mathrm{m}$. (H) Quantitation of lung tissue Areg mRNA expression and Areg levels in BAL fluids. Qualitative data are representative of 3 separate experiments. Dot plots indicate mean \pm SD of $6-10$ replicates from 3 independent experiments. ${ }^{*} P<0.05$ compared with corresponding controls, by 2-way ANOVA.

after siRNA silencing of DUOX1 (Figure 1E). Similarly, dose-dependent activation of EGFR and Src in cultured mouse tracheal epithelial cells (mTECs) in response to Areg was attenuated in mTECs from Duox $1^{-/-}$mice compared with WT mice, which was closely associated with corresponding changes in cysteine oxidation within these kinases (Figure 1F). Collectively, these findings indicate that persistent EGFR activation within epithelia of asthmatic subjects involves oxidative mechanisms originating from DUOX1, which can promote EGFR ligand shedding $(23,27)$ and enhance EGFR tyrosine kinase activity by promoting cysteine oxidation with the kinase domain (25).

DUOX1 contributes to oxidative EGFR activation in HDM-induced allergic asthma in mice. To determine whether enhanced airway DUOX1 expression and activation contribute to airway EGFR activation and protein cysteine oxidation in an experimental model of allergic asthma, we subjected C57BL6/J mice to HDM-induced allergic inflammation (28, 29) (Figure 2A) and evaluated lung tissues for DUOX1 expression and phosphorylation and cysteine oxidation of EGFR and Src. As expected (14), we observed significantly increased lung tissue levels of Duox1 mRNA in HDM-treated mice, with only minor changes in Duox2 mRNA (Figure 2B). Accordingly, increased DUOX protein levels were detected within the

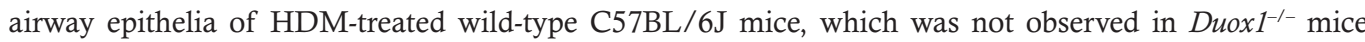
(Figure 2, C and D), confirming that DUOX immunoreactivity originated from DUOX1. To determine whether increased DUOX1 expression was associated with increased overall protein cysteine oxidation during HDM-induced inflammation, lung tissues were derivatized with DCP-Bio1 and the presence of sulfenylated proteins was visualized by streptavidin blotting. Indeed, HDM-treated mice displayed dramatically increased levels of sulfenylated proteins within lung tissues, which was largely prevented in Duox $1^{-1-}$ mice (Figure 2E). Consistent with earlier reports (15) and our observations with NECs, airway EGFR phosphorylation was enhanced during HDM-induced allergic inflammation (Figure 2, F and G), as was phosphorylation of Src (Figure $2 \mathrm{G}$ ), and this was associated with increased cysteine oxidation within these kinases (Figure 2G). HDM-induced EGFR phosphorylation was also associated with enhanced lung tissue mRNA expression of the EGFR ligand Areg and elevated production of AREG in bronchoalveolar lavage (BAL) fluids (Figure 2H). Finally, HDM-induced AREG production, as well as EGFR/Src activation and

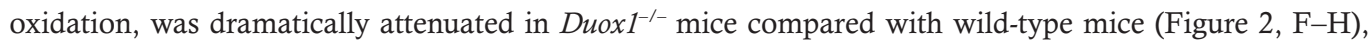
demonstrating the critical contribution of DUOX1 in sustained airway EGFR activation during allergic inflammation. Collectively, these findings indicate that HDM-induced allergic asthma is associated with enhanced DUOX1 expression in the airways and DUOX1-mediated persistent activation of EGFR within the airway epithelium.

DUOX1 contributes to HDM-induced neutrophilic inflammation, mucous metaplasia, subepithelial fibrosis, and airways hyperresponsiveness. HDM-induced airway inflammation was assessed by enumeration of inflammatory cells in the BAL (Figure 3A) and by H\&E staining of lung tissue sections (Supplemental Figure 1A; supplemental material available online with this article; doi:10.1172/jci.insight.88811DS1), which indicated marked inflammation in HDM-challenged mice, with dramatic increases in BAL eosinophils and lymphocytes as well as modest increases in neutrophils. DUOX1 deficiency had minimal effect on the overall inflammatory response and on eosinophil infiltration but dramatically attenuated HDM-induced recruitment of neutrophils (Figure 3A). Airway epithelial EGFR activation has been linked with neutrophilic inflammation in severe human asthma (11) and with HDM-induced neutrophilia in mice (15), suggesting that the observed contribution of DUOX1 to neutrophilic inflammation in the present studies is related to its role in epithelial EGFR activation. Another critical feature of allergic airway inflammation that has been strongly associated with airway epithelial EGFR activation is the development of mucous goblet cell metaplasia $(12,13)$. As expected, HDM-induced allergic inflammation was associated with increased presence of airway mucus, as visualized by PAS staining (Figure 3B), and enhanced lung tissue expression of the mucous metaplasia genes Muc5ac, Clcal, and Postn (Supplemental Figure 1B), and each of these out- 


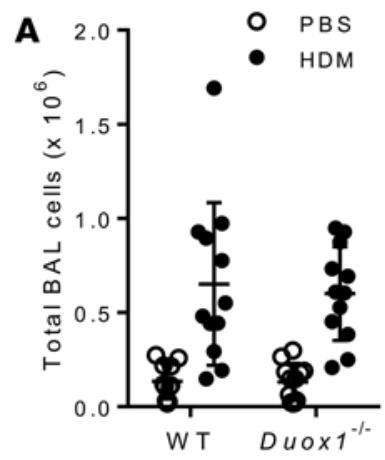

B

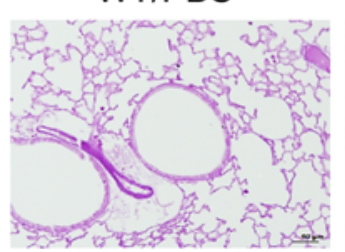

WT/HDM

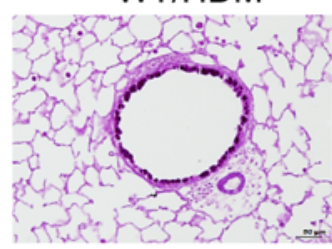

D WT/PBS
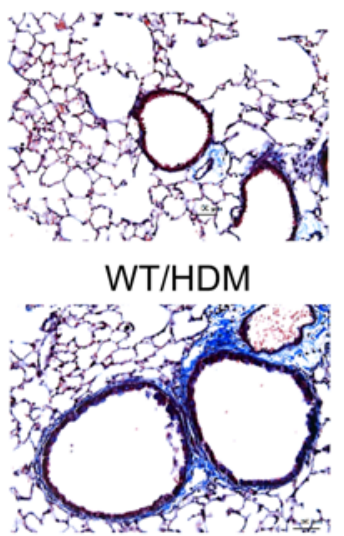

WT/HDM

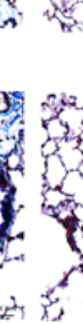

E

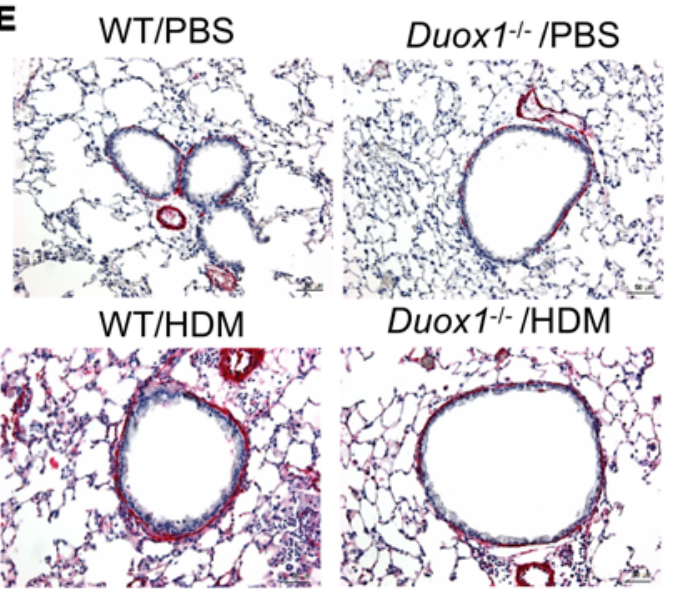

Duox 1 - /PBS

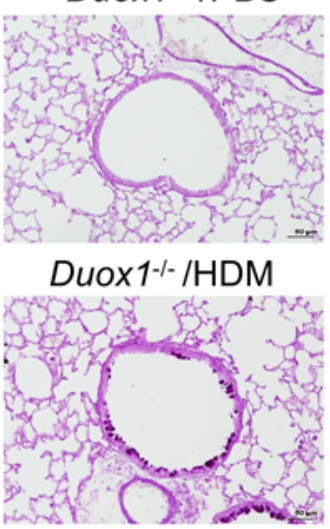

Duox $1 \% / P B S$

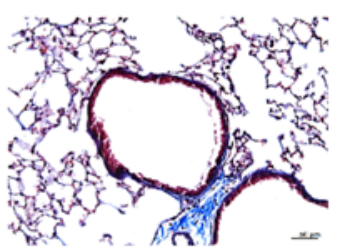

Duox $1^{-/-} /$HDM
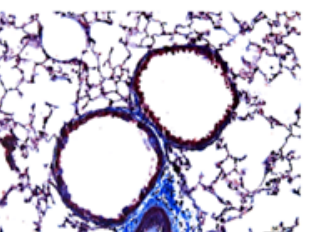

.
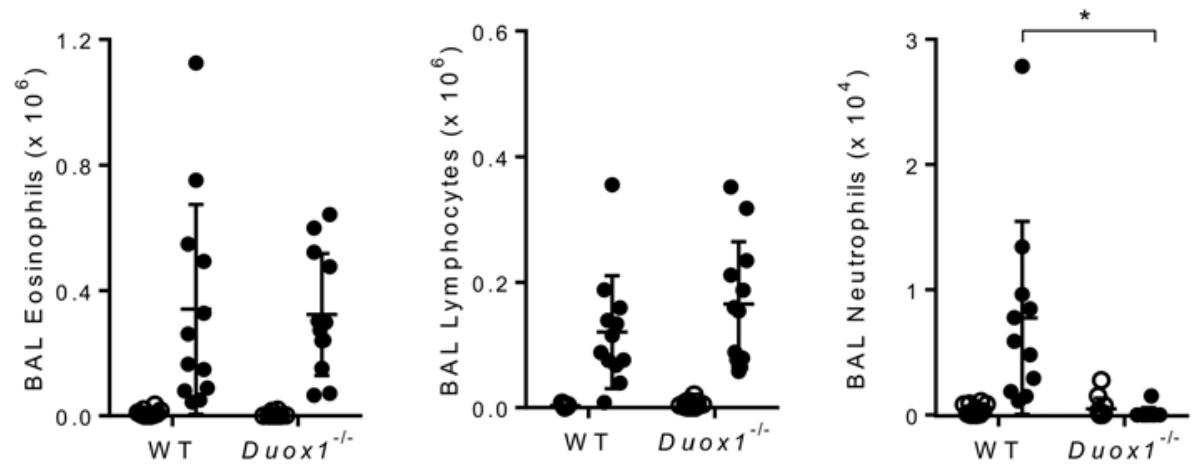

$$
\text { . }
$$

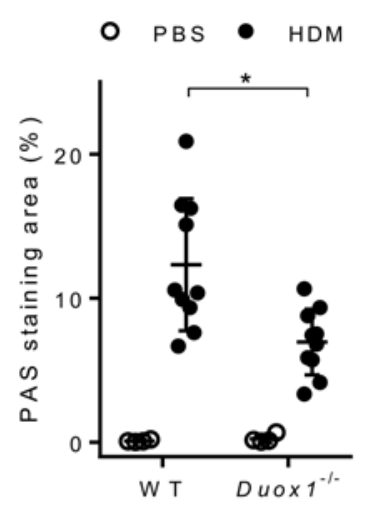

C

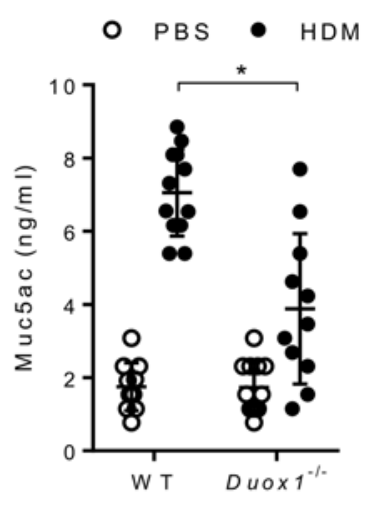

$\mathbf{F}$
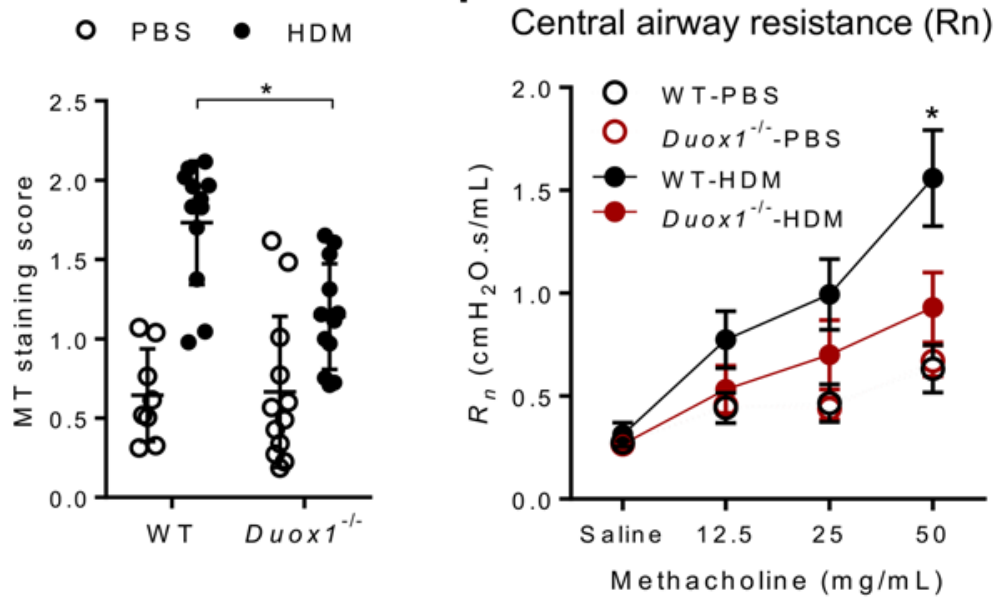

Figure 3. DUOX1 contributes to house dust mite-induced neutrophilic inflammation, mucous metaplasia, subepithelial fibrosis, and airways hyperresponsiveness. (A) Quantification of total and differential cell counts in BAL fluids. (B) Analysis of mucous metaplasia by histochemical analysis of PAS staining and quantification of positive staining areas using Metamorph software. (C) Quantification of Muc5ac protein in BAL fluids by ELISA. (D and E) Evaluation of subepithelial fibrosis by Masson's trichrome staining (D) or $\alpha$-SMA immunostaining (E), as quantified by staining score or percentage of staining around airways. Scale bars: $50 \mu \mathrm{m}$. (F) Analysis of airways central airway resistance $\left(R_{\mathrm{n}}\right)$ in response to methacholine challenge. Representative histochemical images are shown, and dot plots represent mean \pm SD of 8-12 replicates from 3 independent experiments. ${ }^{*} P<0.05$ compared with corresponding controls, using 2-way ANOVA. 
comes was significantly attenuated in Duox $1^{-/-}$mice (Figure 3B and Supplemental Figure 1B). Moreover, HDM-induced mucus production was also demonstrated by increased levels of Muc5ac protein in the BAL, which were also significantly attenuated in Duox $1^{-1-}$ mice (Figure 3C).

In addition to promoting mucous metaplasia, epithelial EGFR activation has also been implicated in allergen-induced subepithelial fibrotic remodeling $(15,16)$. In agreement with this, HDM-induced inflammation was associated with significant subepithelial collagen deposition around peripheral airways, as detected by Masson's trichrome staining (Figure 3D) and accumulation of $\alpha$-smooth muscle actin ( $\alpha$-SMA) (Figure 3E), and induced lung tissue expression of the collagen genes Colla1 and Col3a1 (Supplemental Figure 1B), and each of these outcomes was significantly reduced in Duox $1^{-/}$mice (Figure $3 \mathrm{E}$ and Supplemental Figure 1B). Finally, HDM treatment also resulted in enhanced airway hyperresponsiveness to methacholine, as shown by increased resistance of the conducting airways $\left(R_{\mathrm{n}}\right)$, which was also significantly attenuated in Duox $1^{-/-}$mice (Figure $3 \mathrm{~F}$ and Supplemental Figure 1C). Collectively, these findings indicate that epithelial DUOX1 mediates a number of critical features of allergic airway inflammation, most notably neutrophilia, mucous metaplasia, subepithelial fibrosis, and airways hyperresponsiveness. Since each of these outcomes have in previous studies been linked with enhanced epithelial activation of EGFR $(12,15,16)$, our findings are consistent with the hypothesis that DUOX1 contributes to these phenotypes largely by promoting persistent EGFR activation within the airway epithelium.

DUOX1 participates in HDM-induced neutrophil chemokine and type 2 cytokine production from epithelia and ILC2s. DUOX1-dependent EGFR activation can promote airway epithelial production of the neutrophil chemokine IL-8 $(27,30)$. Analysis of BAL fluids from HDM-challenged wild-type mice indicated marked and DUOX1-dependent increases of the mouse functional IL- 8 homolog CXCL1, consistent with the observed DUOX1-dependent neutrophilia in this model (Figure 4A). In addition, DUOX1 was also found to be critical for HDM-induced increases in lung tissue mRNA levels of Cxcl1 (Supplemental Figure 2A).

We recently highlighted a critical role of DUOX1 in epithelial IL-33 secretion and induction of type 2 cytokines in response to acute challenge to allergens such as HDM (14). In agreement with these findings, BAL fluids from HDM-challenged mice demonstrated increased levels of IL-33, as well as the type 2 cytokines IL-5 and IL-13 (Figure 4B), which were associated with corresponding increases in lung tissue mRNA levels (Supplemental Figure 2B), and each of these responses was dramatically suppressed in HDM-treated Duox $1^{-1-}$ mice. Serum levels of HDM-specific IgG1 and IgE indicated were highly similar between HDM-challenged wild-type and Duox $1^{-/-}$mice (Supplemental Figure 2C), suggesting that DUOX1-dependent type 2 responses are largely independent of adaptive immune responses and are most likely related to expansion and activation of ILC2s, which could serve as the primary source of IL-5, IL-3, and the EGFR ligand AREG in response to epithelial-derived cytokines, such as IL-33, within the lung (31-33). To address this, we generated single-cell suspensions of lung tissues from both control and HDM-challenged mice for in vitro restimulation with IL-33, which revealed dramatically enhanced production of IL-5, IL-13, and Areg by lung cell suspensions from HDM-challenged wild-type mice but not from Duox $1^{-/-}$mice (Figure 4C). These findings would suggest that HDM-induced recruitment of lung ILC2s is attenuated in Duox $1^{1^{-1}}$ mice, but analysis of lung tissue ILC2 populations using flow cytometry analysis of lineage-negative, $\mathrm{CD}^{+} 0^{+}$Ly6A (Sca-1) $)^{+}$cell populations (Supplemental Figure 3) did not demonstrate reduced lung ILC2 populations in HDM-exposed Duox $1^{-/-}$mice compared with HDM-challenged wild-type mice (Figure 4, $\mathrm{D}$ and E); in fact, increased ILC2 recruitment in Duox $1^{1^{-1}}$ mice was indicated. Therefore, DUOX1 may be involved more directly in ILC2-dependent production of type 2 cytokines and AREG. To test this, lung ILC2 populations were expanded in wild-type and Duox $1^{-1-}$ mice by repeated intranasal IL-33 exposure (32) and collected by flow sorting for analysis of DUOX1 protein by Western blot as well as for analysis of Il5, Ill3, and Areg mRNA expression as specific markers of ILC2 development. IL-33-mediated ILC2 recruitment was comparable between WT and Duox $1^{-/-}$mice, but ILC2s from IL-33-challenged Duox $1^{-1-}$ mice expressed markedly reduced mRNA levels of Il5, Il13, and Areg compared with ILC2s from wild-type mice (Figure 4F). Moreover, lung ILC2s were found to express DUOX1 protein (Figure 4G), suggesting that the observed reduction of HDM-induced type 2 responses in Duox $1^{-1-}$ mice is at least partially due to attenuated DUOX1-mediated activation of ILC2s. Type 2 cytokines and AREG can also be produced by airway epithelial cells $(34,35)$, and in vitro stimulation of isolated mTECs with IL-33 produced significant amounts of IL-13 and AREG indeed; these responses were markedly attenuated in mTECs from Duox $1^{-1-}$ mice (Supplemental Figure 2D). In aggregate, these findings establish that DUOX1 is pivotal for epithelial production of CXCL1 and IL-33 but also mediates subsequent activation of type 2 cytokines, such as IL-13 
A $\bigcirc$ PBS $\bullet$ HDM

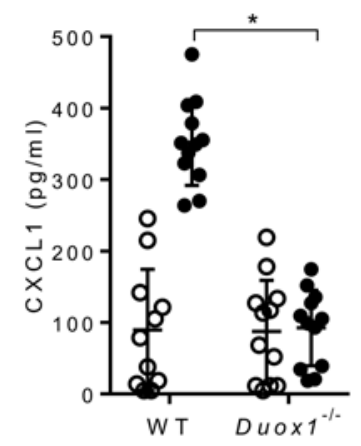

C

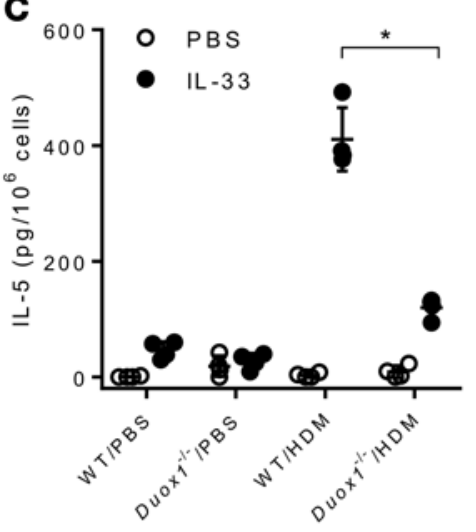

D

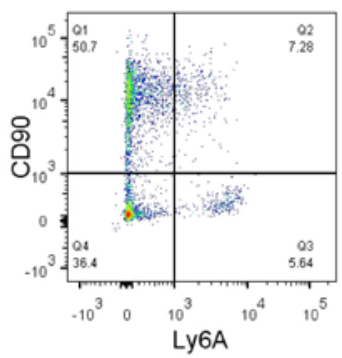

E

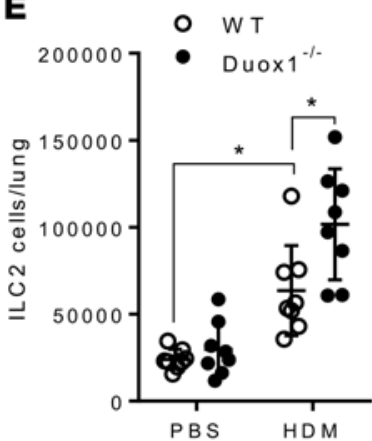

G

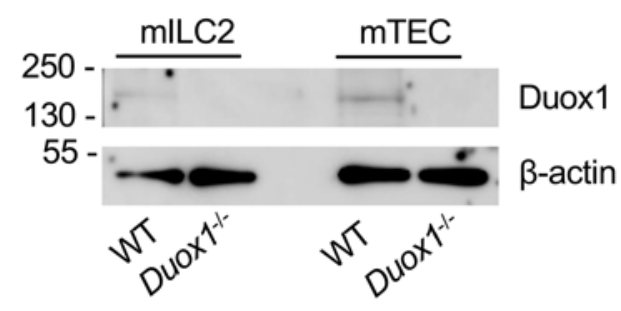

Duox $1^{-/ /} / \mathrm{PBS}$
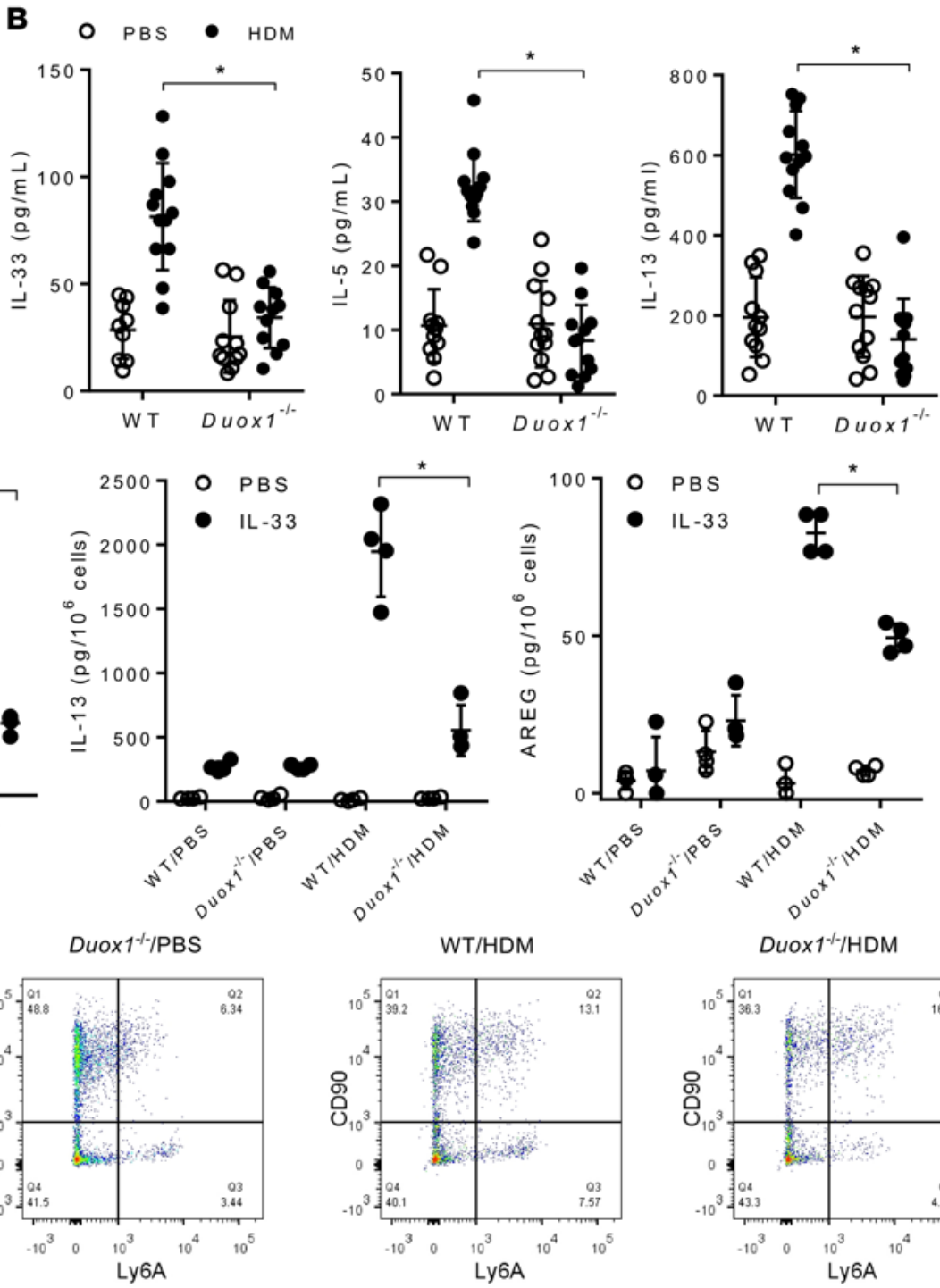

$\begin{array}{ll}\text { - } & \text { PBS } \\ \text { - } & \text { IL }-33\end{array}$

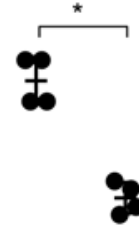

9
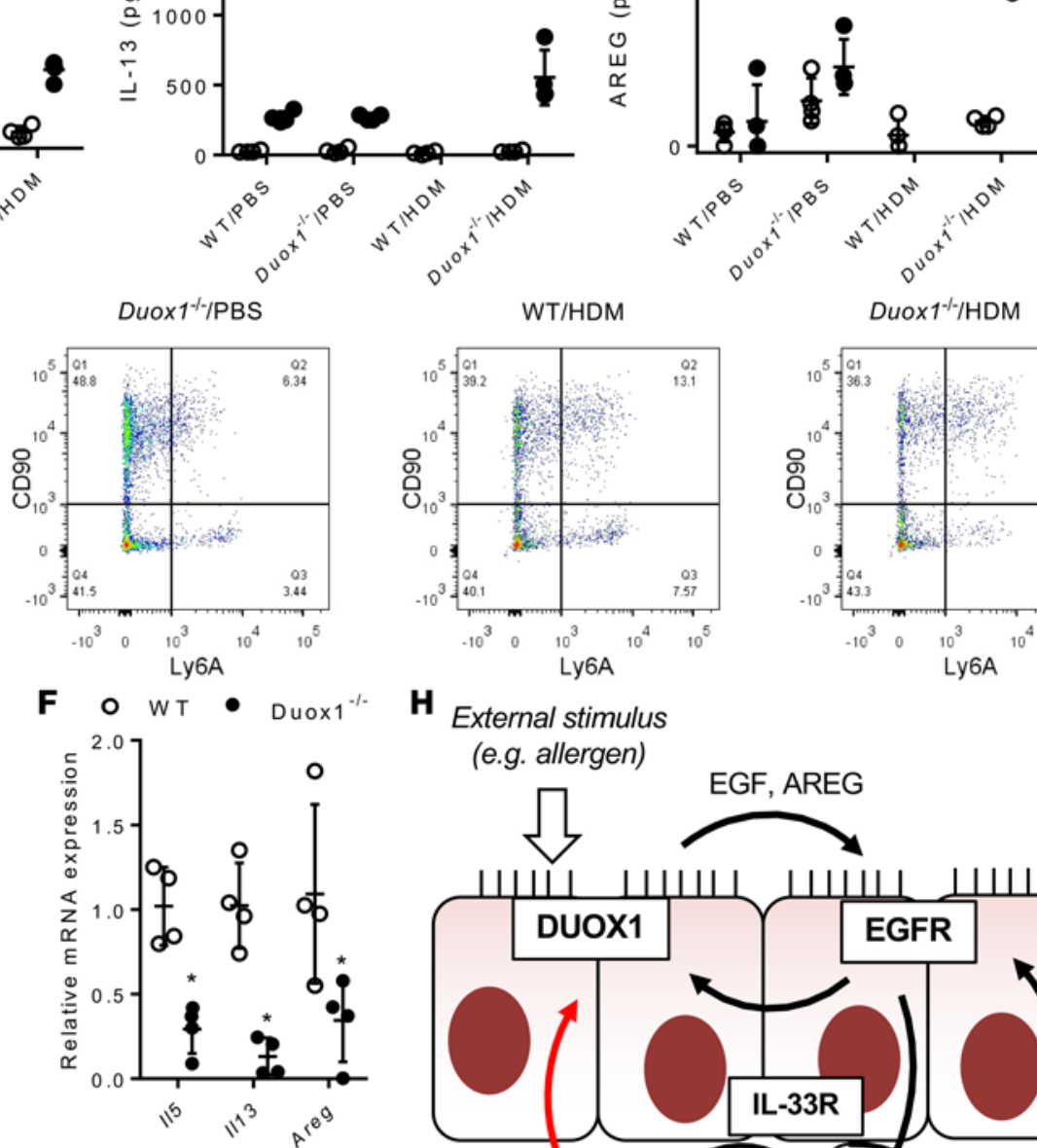

H External stimulus

(e.g. allergen)
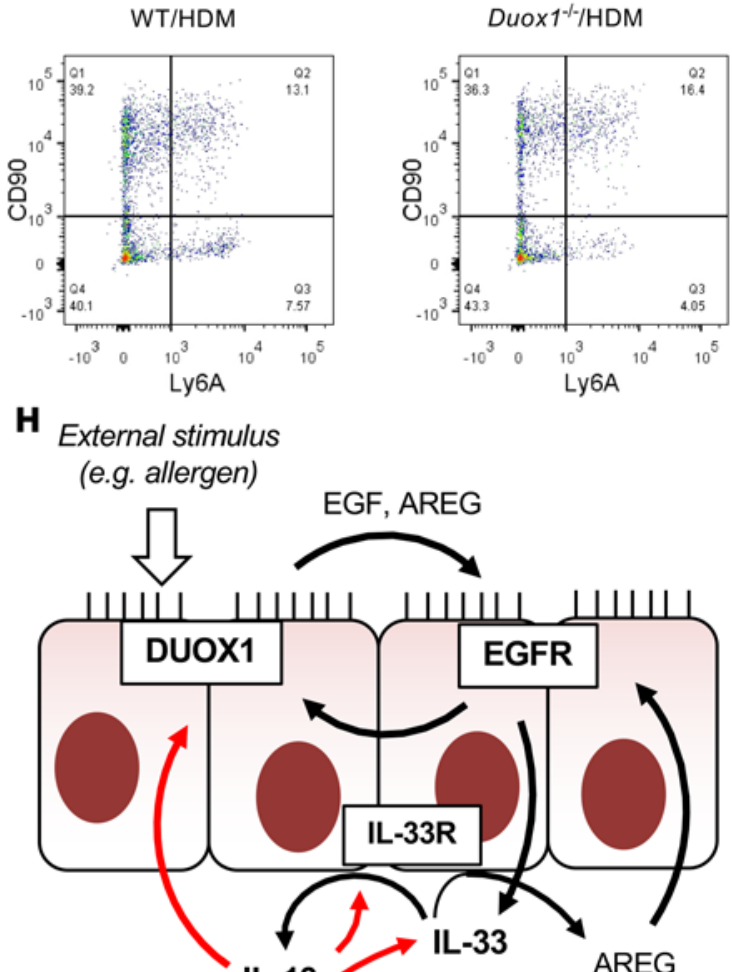

IL-13

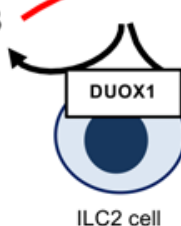


Figure 4. DUOX1 mediates type 2 cytokine responses and ILC2 activation during house dust mite-induced inflammation. (A and B) Analysis of BAL cytokine levels of CXCL1 (KC) (A) and of type 2 cytokines, IL-33, IL-5, and IL-13 (B). (C) Analysis of IL-33-induced (10 ng/ml) production of ILC2-specific cytokines (IL-5, IL-13) and AREG from lung single-cell suspensions of PBS- or HDM-treated mice. (D and E) Flow cytometry analysis of ILC2 populations in lung tissues. (F) RT-PCR analysis of flow-sorted lung ILC2 populations from IL-33-triggered mice for type 2 cytokines, Areg, and Duox1. (C) Representative western blot analysis of Duox1 protein and $\beta$-actin in sorted ILC2 cells (mILC2) and primary mTECs. (H) Schematic representation of proposed interactions among DUOX1, EGFR, IL-33, and IL-13 within the airway. Black arrows reflect enzyme activation or stimulation of cytokine secretion, and red arrows indicate effects on mRNA expression. Dot plots represent mean \pm SD of 8-12 replicates from $2-3$ separate experiments. ${ }^{*} P<0.05$ compared with corresponding controls by 2 -way ANOVA.

and AREG, by both epithelial cells and ILC2s (Figure 4G). Collectively, these events contribute to the observed persistent epithelial activation of EGFR, and enhanced neutrophilia, mucous metaplasia, and fibrotic remodeling, during HDM-induced allergic inflammation.

DUOX1 targeting during ongoing allergic inflammation attenuates EGFR activation, mucous metaplasia, and fibrotic remodeling. While these data establish that DUOX1 contributes markedly to development of several clinically relevant features of allergic asthma, it does not necessarily imply that targeting of DUOX1 can reverse or attenuate these outcomes in ongoing allergic airway disease. To test this, we administered Duox1-targeted siRNA into the airways of mice after establishing HDM-induced allergic inflammation (Figure 5A) to evaluate the ability of DUOX1 suppression to reverse symptoms of ongoing allergic asthma phenotypes or asthma exacerbations. siRNA-dependent DUOX1 targeting was efficacious and significantly reduced Duox1 expression in lung tissues (Supplemental Figure 4A) and markedly attenuated HDM-induced activation and oxidation of EGFR and Src within the lung (Figure 5B). Moreover, siRNA targeting of Duox1 was capable of reversing several critical phenotypes of HDM-induced allergic inflammation, such as neutrophil infiltration (Figure 5C), type 2 cytokine responses (Figure 5D and Supplemental Figure 4B), indices of mucous metaplasia (Figure 5, E and F, and Supplemental Figure 4C), and fibrotic remodeling (Figure 5G and Supplemental Figure 4, C and D). Finally, Duox1 silencing tended to attenuate HDM-induced central airway resistance in response to methacholine challenge, although differences were not statistically significant (Supplemental Figure 4D). Collectively, these findings indicate that DUOX1 targeting in a model of previously established asthma is capable of reversing several clinical relevant asthma features.

To date, no specific pharmacological inhibitors of DUOX1 are available. While several drug screening and development efforts have identified inhibitors with some specificity against several NADPH oxidase isoforms, none of these have been tested for efficacy against DUOX1 (36). We performed preliminary analysis of several putative NADPH oxidase inhibitors to evaluate their ability to inhibit DUOX1 activation in HBE1 cells, which was assessed by analysis of ATP-stimulated extracellular $\mathrm{H}_{2} \mathrm{O}_{2}$ production and IL-33 secretion $(14,23)$. Both ATP-stimulated $\mathrm{H}_{2} \mathrm{O}_{2}$ production and IL-33 secretion were inhibited in a dose-dependent fashion by the nonselective NADPH oxidase inhibitor diphenylene iodonium (DPI) as well as ML171, a phenothiazine compound that was recently identified in a high-throughput drug screen as a relatively selective NOX1-selective inhibitor but was not tested against DUOX1 (37), with $\mathrm{IC}_{50}$ values in the order of $0.1-1 \mu \mathrm{M}$ (Supplemental Figure 5, A and B). By comparison, two other NOX inhibitors, apocynin and VAS2870 (36), were relatively ineffective at concentrations up to $10 \mu \mathrm{M}$ (Supplemental Figure $5 \mathrm{~A}$ ). We evaluated the ability of DPI or ML171 to attenuate intrinsic EGFR activation and oxidation in cultured NECs from asthmatic subjects and found that both inhibitors consistently suppressed basal phosphorylation and oxidation of both EGFR and Src in NECs from at least 3 separate asthmatic subjects (Figure 6A). We extended these findings by intranasal administration of either inhibitor into the airways of mice with ongoing HDM-induced allergic inflammation (Figure 6B), which similarly suppressed oxidation and activation of both EGFR and Src (Figure 6C). Administration of DPI and ML171 did not significantly alter HDM-induced inflammatory BAL cell counts, although they tended to suppress inflammation (Supplemental Figure 5C) and to attenuate methacholine-induced central airway resistance in HDM-treated mice (Supplemental Figure 5D). Moreover, both inhibitors significantly suppressed production of type 2 cytokines (Figure 6D and Supplemental Figure 5E) as well as indices of mucous metaplasia and fibrotic remodeling (Figure $6 \mathrm{E}$ and Supplemental Figure $5 \mathrm{~F}$ ). The close association of these outcomes with DUOX1 activation suggests that these inhibitors acted largely by inhibiting DUOX1, although inhibitory actions on other NOX isozymes may have contributed as well. Collectively, these results demonstrate that pharmacological targeting of DUOX1 in the airways is potentially clinically useful as a therapeutic approach to minimize persistent airway EGFR activation and consequent neutrophilia, mucous metaplasia, and fibrotic remodeling, which are especially important in severe asthma. 
A

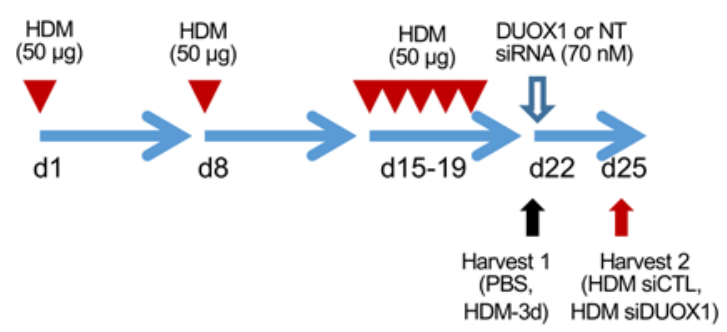

C

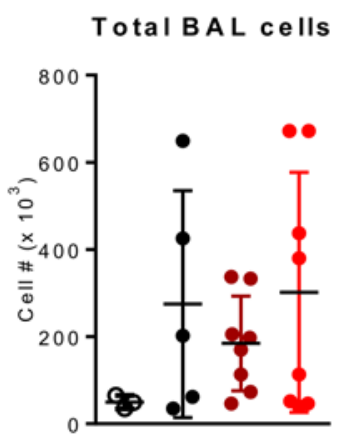

Neutrophils

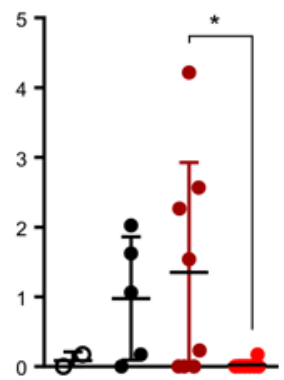

B

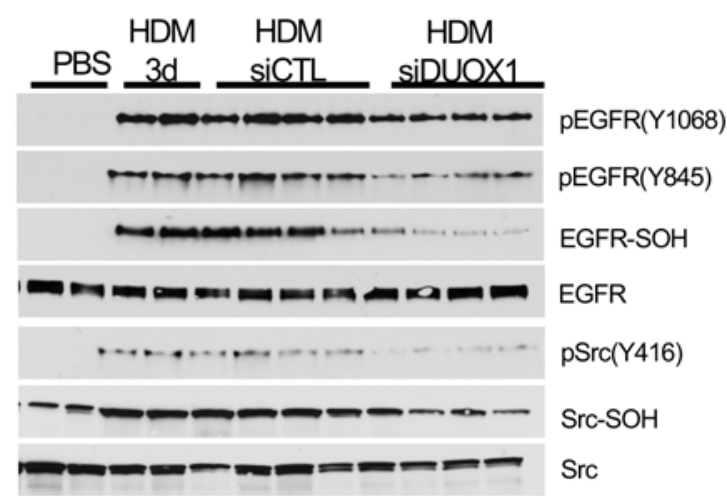

○ PBS - HDM-sictl

- HDM-3d HDM-siduox1

D

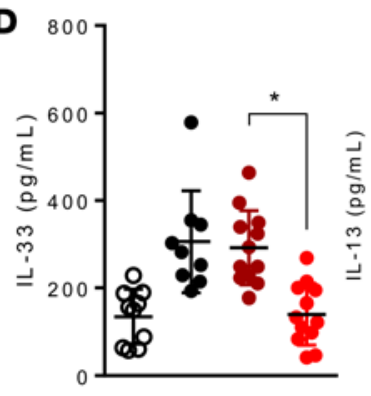

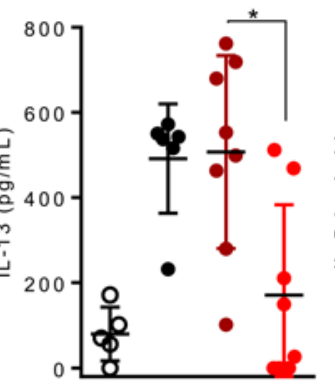
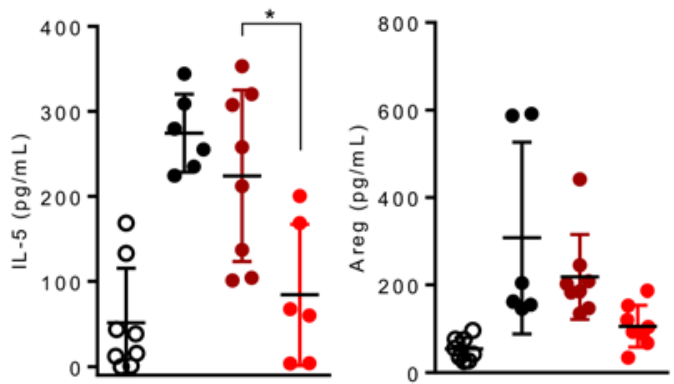

E
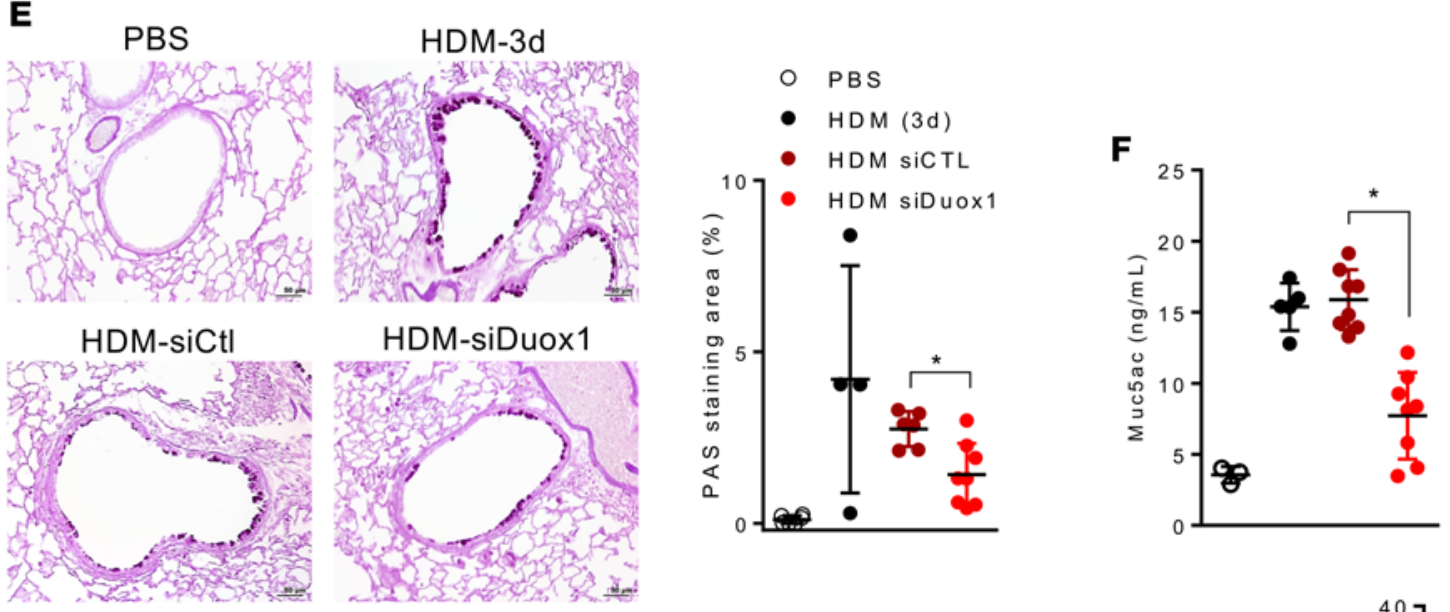

G

PBS

HDM-3d

HDM-siCtl

HDM-siDuox1
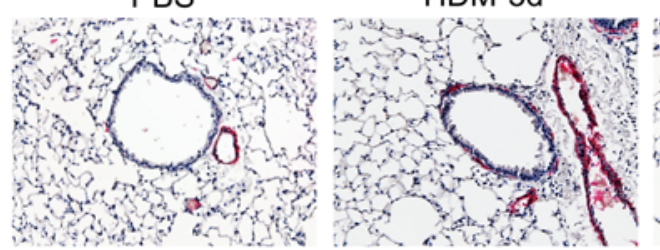

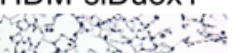
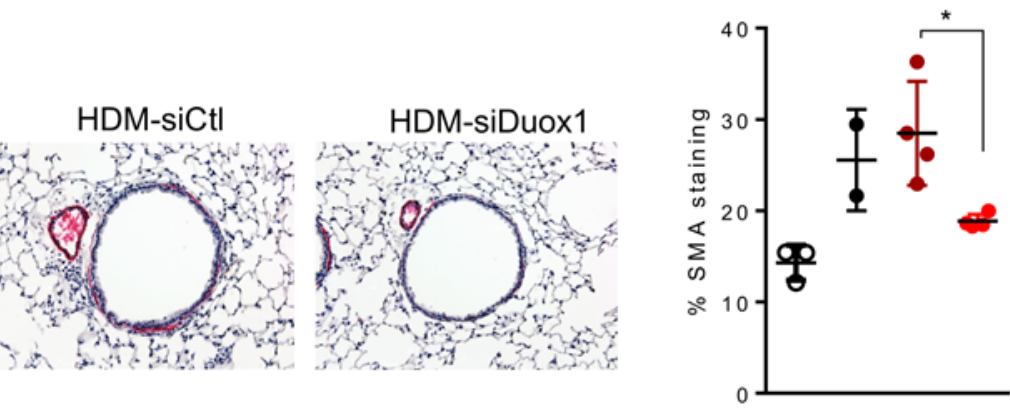
Figure 5. DUOX1 targeting reverses house dust mite-induced EGFR activation, type 2 immune responses, and airway remodeling. (A) C57BL/6) mice were subjected to house dust mite-induced (HDM-induced) inflammation and were subsequently targeted with DUOX1 siRNA. (B) Western blot analysis of EGFR and Src activation and cysteine oxidation, as in Figure 2. (C) Quantitation of BAL total cell counts and neutrophils. (D) ELISA analysis of BAL type 2 cytokines and AREG. (E and F) Evaluation of mucous metaplasia by PAS staining (E) and Muc5ac ELISA of BAL fluids (F). (C) Evaluation and quantification of $\alpha$-smooth muscle actin (SMA) immunoreactivity. Scale bars: $50 \mu \mathrm{m}$. White and black dots represent analysis at day 22 ( 3 days after final HDM challenge), and red dots reflect analysis at day 25 ( 6 days after final HDM and 3 days after siRNA instillation). Dot plots represent mean \pm SD of $6-10$ replicates from 2 separate experiments. ${ }^{*} P<0.05$ compared with corresponding controls, by 2 -way ANOVA.

\section{Discussion}

The most significant findings of the studies presented here are that the epithelial NADPH oxidase DUOX1 regulates several clinically important features of allergic asthma and that DUOX1 targeting is capable of reversing features of ongoing allergic inflammation. While DUOX1-dependent redox signaling may potentially affect many diverse pathways (e.g., refs. 38, 39), the contribution of DUOX1 to allergic inflammation was found to be closely related to its role in persistent oxidant-dependent epithelial activation of EGFR and consequent production of neutrophil chemokines and type 2 cytokines such as IL-13, which results in promotion of neutrophilic inflammation, mucous metaplasia, subepithelial fibrotic remodeling, and central airway resistance. The importance of airway EGFR activation to these various features of allergic asthma is well recognized $(11,15,17)$, but existing EGFR tyrosine kinase inhibitors have not been evaluated for clinical application in chronic lung diseases such as asthma, because of the broad role of EGFR in various organ systems and reported adverse effects that are tolerable in severe or fatal lung diseases, such as lung cancer, but not in lung diseases that are not immediately life-threatening (12, 18-20). The fact that DUOX1 expression is largely restricted to epithelial lineages (39), including the lung, renders it a more appealing therapeutic target, with the potential of avoiding unwanted side effects associated with EGFR inhibitors administered systemically. Our findings indicate that inhibition of DUOX1 may be capable of disrupting the persistent cycle of EGFR activation and activation of type 2 cytokines such as IL-13, which sustain mucous cell metaplasia and fibrotic remodeling within the asthmatic airway. Moreover, we present evidence that DUOX1 targeting in mice with previously established allergic airway inflammation and remodeling can attenuate these outcomes. To our knowledge, our findings are the first to establish the causal participation of DUOX1 in any disease pathology. Related findings were recently observed in mice deficient in Duoxa genes, resulting in overall functional DUOX1/2 deficiency, which similarly showed lack of neutrophilia, type 2 cytokine responses, and mucous cell metaplasia in an alternative model of allergic asthma (40); however, these studies did not address the specific roles of either DUOX1 or DUOX2, which have distinct and nonredundant functions in various organ systems $(38,39)$. Our present findings therefore provide a strong incentive for the development of DUOX1-targeted pharmacological approaches for clinical use, as DUOX1 has so far been relatively ignored in screening efforts for NOX-selective inhibitors (36).

Goblet cell hyperplasia and mucus hypersecretion are important features in the conducting airways during allergic asthma, and extensive mucus plugging is associated with fatal asthma episodes (41). Animal models of allergic asthma support the critical role of the mucin gene Muc5ac in the development of mucus obstruction and airway hyperreactivity (42). The process of mucus hypersecretion during allergic asthma involves a complex multistep process, including mucin gene induction, mucus packaging in storage granules, mucus secretion, and changes in extracellular mucus viscosity. Both EGFR and IL-13 are known to contribute to mucous cell metaplasia by promoting mucin gene induction (e.g., MUC5AC, CLCA1) through independent and still incompletely understood mechanisms $(43,44)$. The present findings indicating a role for DUOX1 in induction of various genes associated with mucous cell metaplasia ( $M u c 5 a c$, Clca1, Postn) are consistent with previous observations (22) and are in keeping with the presence of DUOX1 in the central airways and the observed enhanced central airway resistance in response to methacholine. Enhanced IL-13 production during allergic asthma has also been strongly associated with airway remodeling and subepithelial fibrosis by incompletely understood mechanisms that include alternative macrophage activation, induction of collagen deposition, and myofibroblast proliferation (41). The observed contribution of DUOX1 to subepithelial fibrosis is most likely a result of its importance in IL-13 production by both epithelial cells and ILC2s. Recent development of various antibody-based targeting strategies against IL-13 and its receptor have been met with mixed success (41), and because of the excessive costs of such antibody-based approaches, pharmacologic targeting of DUOX1 with small-molecule inhibitors to minimize IL-13 production might represent an attractive and potentially equally effective alternative. 
A

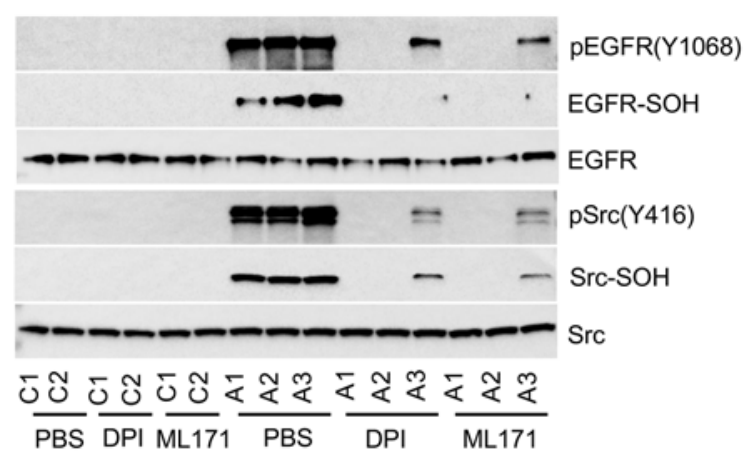

B

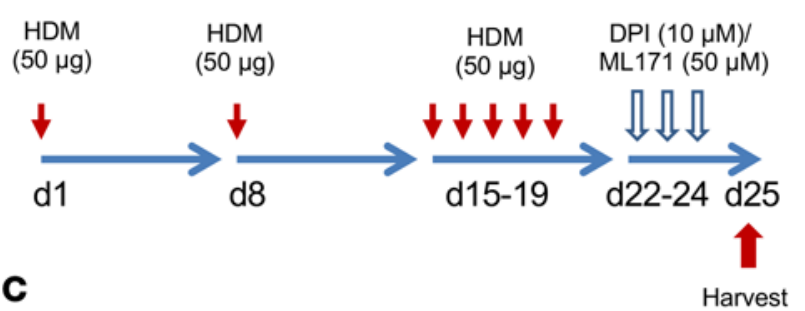

D
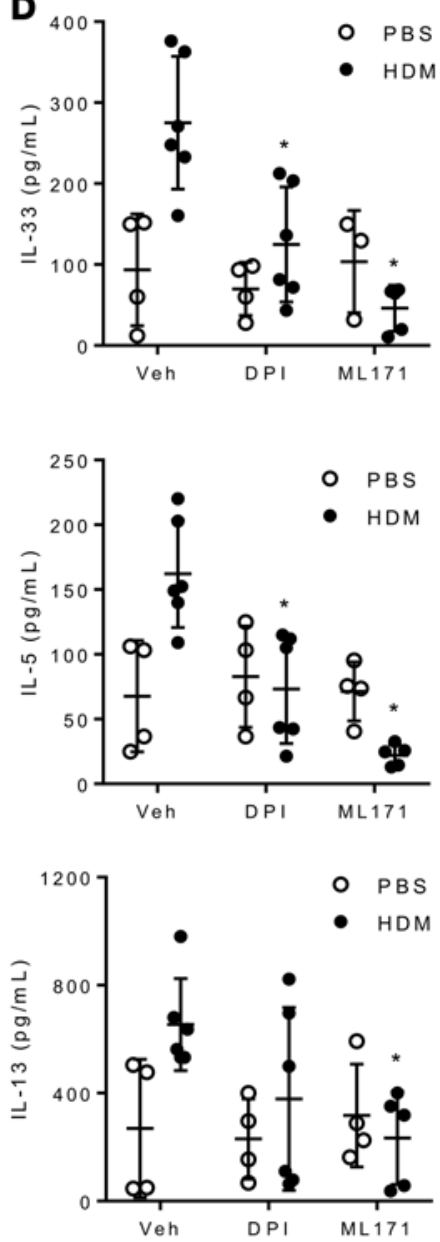

E
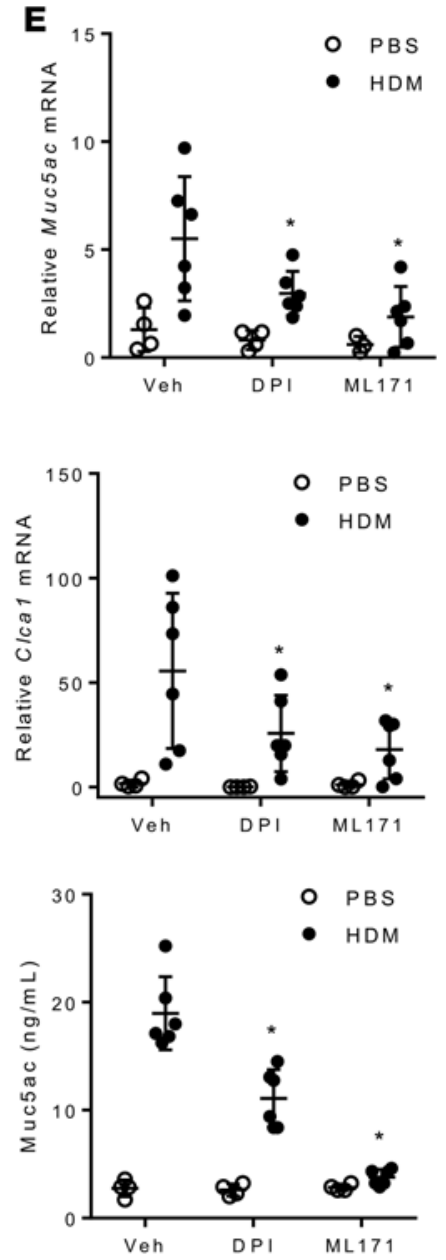

PBS

HDM

Figure 6. Pharmacological NOX inhibition suppresses EGFR activation and type $\mathbf{2}$ immune responses in asthmatic airways. (A) Western blot analysis of activation and cysteine oxidation of EGFR and Src in NECs from 2 healthy (C1, C2) and 3 asthmatic subjects (A1-A3), after 30 minutes of preincubation with the NOX inhibitors DPI $(1 \mu \mathrm{M})$ or ML171 $(5 \mu \mathrm{M})$. (B) C57BL/6) mice were subjected to house dust mite-induced (HDM-induced) allergic inflammation and subsequently administered DPI or ML171 and harvested at day 25 (6 days after final HDM challenge). (C) Western blot analysis of activation and cysteine oxidation of EGFR and Src in lung tissues collected at day 25. (D) ELISA analysis of type 2 cytokines in BAL fluids, harvested at day 25. (E) Evaluation of mucous metaplasia markers in lung tissues by RT-PCR and Muc5ac ELISA of BAL fluids, harvested at day 25. Representative Western blots are shown. Dot plots represent mean \pm SD of 6-10 replicates from 2 separate experiments. ${ }^{*} P<0.05$ compared with corresponding controls, by 2 -way ANOVA.

Our findings highlight the complex and reciprocal interactions of DUOX1 with both EGFR activation and type 2 cytokine signaling (IL-33, IL-13), both well-known features of allergic asthma, and indicate that DUOX1 may contribute to sustained activation of these pathways through several positive feedback loops (Figure 4H). First, DUOX1 critically contributes to EGFR activation in response to external damaging stimuli, including allergens, by promoting epithelial IL-33 secretion and subsequent activation type 2 cytokine responses (IL-5, IL-13) in ILC2s, for example, as well as other lung cell types (14). In turn, IL-13 is capable of enhancing epithelial EGFR activation and inducing epithelial expression of DUOX1 (45) as well as IL-33 and its receptor (IL33R, also known as ST2) (46), thus generating positive feedback loops that result in amplified and prolonged epithelial responses to allergen challenge. An additional consequence of IL-33-mediated ILC2 recruitment and activation is the production of AREG, which forms an essential component of EGFR-mediated epithelial repair responses in the context of parasite/helminth infection (47) but may also contribute to sustained EGFR activation in chronic asthma. Moreover, DUOX1 contributes to EGFR activation not only by promoting EGFR ligand activation, as previously reported $(23,27)$, but also by promoting ligand-induced activation of EGFR phosphorylation by direct cysteine oxidation within EGFR. Although chronic epithelial alterations during allergic asthma are commonly attributed to epigenetic changes, potentially involving important asthma-relevant cytokines such as IL-13 $(8,48,49)$, our 
results indicate that such alterations are also mediated by redox-dependent mechanisms due to enhanced expression and activation of DUOX1 and can be attenuated by inhibition of DUOX1.

Although several EGFR ligands are known to be activated in the airways of asthmatic subjects, the relative contribution of these ligands in disease pathology is unclear. In this light, it is intriguing that AREG differs from other EGFR ligands such as EGF in that it has lower receptor affinity and thus results in sustained EGFR activation, in contrast to rapid EGFR internalization and degradation by other EGFR ligands, with distinct cellular consequences (47). Elevated AREG levels are detected in asthmatic airways, especially after exacerbations (9), and AREG appears to be the most strongly upregulated EGFR ligand in NECs from asthmatics (according to the present studies) and is also a major product of activated ILC2s. These observations suggest that AREG is primarily responsible for the observed sustained EGFR activation in asthmatic airways. Moreover, AREG may also contribute to mucous cell metaplasia in some conditions, although its contribution to mucous cell metaplasia in the context of allergic asthma is less clear (50). Although the EGF ligands responsible for the various asthma phenotypes need to be further unraveled, DUOX1 is clearly involved in EGFR activation irrespective of the activating ligand.

The interactions among DUOX1, EGFR, and IL-33/IL-13 responses are even further complicated by our unanticipated finding that DUOX1 also participates in IL-33-dependent activation of type 2 responses in epithelial cells as well as ILC2s. The mechanism by which DUOX1 promotes IL-33-dependent signaling will need to be addressed in future studies but potentially involves amplification of IL33R-dependent JAK/STAT signaling by oxidative inhibition of protein tyrosine phosphatases, analogous to other cytokine signaling pathways (51). Moreover, the observed expression of DUOX1 in ILC2s, combined with recent reports indicating the presence of DUOX1 in T cells (51) and macrophages (52), implies that the involvement of DUOX1 in lung biology extends beyond its well-studied role(s) in epithelial host responses (53) and likely also includes regulatory functions in other cell lineages. Future studies, using transgenic models of targeted DUOX1 deletion in these various cell lineages, for example, will be required to unravel the specific contribution of DUOX1-dependent signaling in these specific cell types.

The critical function of DUOX1 in induction of type 2 cytokine responses (including IL-5) during HDM-induced inflammation appears to be at odds with the relative lack of impact of DUOX1 on HDM-induced eosinophilia. One potential explanation for this may be the fact that ILC2 can produce IL-5 constitutively to control eosinophil homeostasis (54), whereas IL-13 production requires ILC2 stimulation by IL-33 and/or IL-25 (55). Indeed, observations of enhanced accumulation of ILC2 populations during HDM-induced inflammation in DUOX1-deficient mice would suggest that constitutive IL-5 production by these ILC2s may have been sufficient to promote eosinophil recruitment, even though IL-33-mediated induction of IL-5 is impaired in these cells.

Our studies are primarily based on the premise that DUOX1 activation generate $\mathrm{H}_{2} \mathrm{O}_{2}$ as its primary product and that $\mathrm{H}_{2} \mathrm{O}_{2}$ affects protein function by cysteine oxidation to sulfenic acids and related oxidative modifications such as $S$-glutathionylation $(38,39)$. Although the present studies focused primarily on sulfenylation as a mechanism of oxidative EGFR activation, based on recent findings $(25,56)$, we cannot rule out the potential contribution of protein glutathionylation within EGFR or in other bystander proteins in mediating DUOX1-related allergic inflammation (e.g., ref. 57, 58). Likewise, it is plausible that $\mathrm{H}_{2} \mathrm{O}_{2}$ gives rise to production of other (secondary) reactive intermediates that would expand the range of potential oxidative posttranslational modifications that affect protein function. Although such additional mechanisms cannot be ruled out, our present findings indicate that oxidant-mediated activation of EGFR signaling represents a major mechanism by which DUOX1 contributes to allergic airway inflammation and remodeling.

In summary, the findings presented here highlight a critical function of airway DUOX1 in promoting epithelial EGFR activation and subsequent cytokine responses and airway remodeling during allergic asthma and furthermore demonstrate that therapeutic targeting of DUOX1 has the potential of reversing these outcomes in cases of severe asthma and potentially in cases of exacerbations. Although the pharmacological NOX inhibitors used in this study may not be suitable for clinical development (36), they strongly support the proof of concept that selective targeting of DUOX1 may have excellent therapeutic potential.

\section{Methods}

Study design. This study was designed to address the importance of increased DUOX1 expression in the airways of subjects with allergic asthma and its role in mediating EGFR activation as a major feature of epithelial alterations that drive type 2 inflammation and airway remodeling (14). Primary human NECs were 
collected from a cohort of 17 healthy volunteers and 16 subjects with allergic rhinitis and asthma (14). Atopy was confirmed by positive skin tests and elevated serum $\operatorname{IgE}(>100 \mathrm{IU} / \mathrm{ml})$, and asthma was confirmed by positive response to bronchodilator ( $\geq 200 \mathrm{cc}$ and $12 \%$ improvement in FEV1 and/or FVC) or a positive methacholine challenge test $\left(\mathrm{PC}_{20}<8 \mathrm{mg} / \mathrm{ml}\right)$. Healthy volunteers had no history of rhinitis or asthma, negative skin tests, and negative methacholine challenge tests. Cultured NECs or bronchial epithelial HBE1 cells (59) were analyzed for protein cysteine oxidation and EGFR activation, and the role of DUOX1 was determined using genetic or pharmacologic inhibitor approaches. The importance of DUOX1 in allergic asthma was also evaluated in a mouse model of HDM-induced allergic inflammation using both male and female C57BL/6J wild-type mice (The Jackson Laboratory) and DUOX1-deficient mice, originally generated using a retroviral-based gene-trapping method (Lexicon Pharmaceuticals) and backcrossed onto a C57BL/6J background (60). In addition, the therapeutic potential of DUOX1 inhibition during ongoing asthma exacerbations was assessed by in vivo oropharyngeal administration of DUOX1-targeted siRNA or pharmacological inhibitors in mice 3 days after establishing HDM-induced allergic inflammation. HDM-induced allergic inflammation and airway remodeling were assessed by immunohistochemical analyses, RT-PCR, cytokine production by ELISA, analysis of airways hyperresponsiveness, and Western blot analysis of EGFR activation and protein cysteine oxidation in lung tissues. Animal studies are based on 2-3 separate experiments with randomized male and female mice and 8-12 mice per experimental group; no outliers were excluded from data analyses.

Isolation and culture of human nasal and bronchoepithelial cells. Primary NECs were collected by gentle stroking of the inferior turbinate surface with a Rhino-Probe curette (Arlington Scientific) and cultured as recently described (61) in bronchial epithelial growth medium (Cambrex Bioscience Walkersville Inc.). For experiments, NECs were plated on collagen-coated 24-well culture plates (Costar) and cultured in a 1:1 mixture of bronchial epithelial cell basic medium and DMEM-H with SingleQuot supplements (Cambrex), bovine pituitary extracts $(13 \mathrm{mg} / \mathrm{ml})$, bovine serum albumin $(1.5 \mu \mathrm{g} / \mathrm{ml})$, and nystatin (20 units). The immortalized bronchial epithelial cell line HBE1 was maintained as described previously $(23,59)$. For in vitro cell studies, cells were grown to confluence in 24-well culture plates (Corning) and incubated overnight in full media without EGF to suppress basal EGFR activity, prior to treatment with indicated stimuli or inhibitors.

Mouse studies. C57BL6/J or Duox $1^{-/-}$mice (aged 8-12 weeks) were subjected to brief isofluorane anesthesia for intranasal administration of $50 \mu \mathrm{g}$ of $\mathrm{HDM}$ extract (D. pteronyssinus; Greer Laboratories; 1.27 endotoxin units/mg) or $50 \mu \mathrm{PBS}$ vehicle control, 5 days/week for 3 weeks, and harvested at 3 days after the final HDM challenge for the various analyses described below. For intervention studies using Duox 1 -targeted siRNA or NOX inhibitors, C57BL6/J mice were sensitized to HDM by intranasal instillation on days 1 and 8, followed by HDM challenge on days 15-19. Three days after the final challenge (day 22), mice were administered Duox1-targeted siRNA (two DUOX1 siRNA targets at $35 \mu \mathrm{g} /$ target sequence/mouse or $70 \mu \mathrm{g}$ NS siRNA, instilled oropharyngeally in mice under brief isofluorane anesthesia) (21), or the NOX inhibitors DPI (10 $\mu \mathrm{M}$, $50 \mu \mathrm{l})$ or ML171 $(50 \mu \mathrm{M}, 50 \mu \mathrm{l}(36,37)$, or appropriate vehicle control, and animals were harvested 3 days later (day 25) for various analyses. At harvest, BAL and lung tissues were collected for the various analysis listed below. mTECs were isolated from excised mouse tracheas from either wild-type mice or DUOX1-deficient mice and cultured as previously described (21) and used at passage 2-3 in in vitro experiments.

siRNA silencing. HBE1 cells were transfected at 60\%-70\% confluence for transfection with Silencer Negative Control \#1 siRNA (Invitrogen) or DUOX1-targeted siRNA: (sense) GCUAUGCAGAUGGCGUGUAtt, (antisense) UACACGCCAUCUGCAUAGCtg. For in vivo siRNA silencing of DUOX1, two DUOX1 siRNA targets in sterile PBS (35 $\mu \mathrm{g} /$ target sequence/mouse) or NS siRNA (70 $\mu \mathrm{g} / \mathrm{mouse}$ ) were instilled oropharyngeally in mice under brief isofluorane anesthesia, as detailed previously (21).

Western blotting. Cell lysates or lung homogenates were prepared using Western solubilization buffer (50 mM HEPES, $250 \mathrm{mM} \mathrm{NaCl}, 1.5 \mathrm{mM} \mathrm{MgCl}$, 1\% Triton X-100, 10\% glycerol, 1 mM EGTA, 1 mM PMSF, $2 \mathrm{mM} \mathrm{Na}_{3} \mathrm{VO}_{4}, 10 \mu \mathrm{g} / \mathrm{ml}$ aprotinin, $10 \mu \mathrm{g} / \mathrm{ml}$ leupeptin; $\mathrm{pH}$ 7.4), and samples containing equal amounts of protein (measured using the BCA protein assay kit; Pierce) were separated on 10\% SDS-PAGE gels, transferred to nitrocellulose membranes, and probed with antibodies against EGFR (C74B9; 1:1,000), pEGFR Tyr845 (D63B4; 1:500), pEGFR Tyr1068 (D7A5; 1:1,000), and Src (L4A1; 1:1,000) (all from Cell Signaling); p-Src Tyr416 (1:1,000; 07-909; Millipore); or DUOX1 (provided by F. Miot, Free University of Brussels, Brussels, Belgium) (62). Primarily antibodies were probed with rabbit- or mouse-specific secondary antibodies conjugated with HRP (Cell Signaling) and detected by chemiluminescence using SuperSignal 
West Pico Chemiluminescent Substrate (Pierce). Quantitative EGFR phosphorylation status was assessed by densitometry analysis of pEGFR/EGFR ratios using ImageJ software (NIH).

Analysis of protein cysteine oxidation. Cells or lung tissues were homogenized in deoxygenated Western solubilization buffer containing $1 \mathrm{mM}$ DCP-Bio1 (Kerafast), $200 \mathrm{U} / \mathrm{ml}$ catalase (Worthington), and 10 $\mathrm{mM} N$-ethyl-maleimide (NEM; Sigma-Aldrich); incubated for 1 hour on ice; and centrifuged (5 minutes, $15,000 \mathrm{~g}$ ) to remove debris. Excess DCP-Bio1 and NEM were removed by 6 successive washes with $20 \mathrm{mM}$ Tris. $\mathrm{HCl}$ ( $\mathrm{pH}$ 7.4) on Amicon Ultra-0.5 Centrifugal Filter Devices (Millipore), and biotin-tagged proteins were subsequently purified using NeutrAvidin agarose beads (by overnight incubation with $50 \mu 1$ of 50:50 slurry; Pierce), followed by successive washes with $1 \%$ SDS, $4 \mathrm{M}$ urea, $1 \mathrm{M} \mathrm{NaCl}$, and $100 \mathrm{mM}$ ammonium bicarbonate (with and without $10 \mathrm{mM}$ DTT) to remove nonspecifically bound proteins $(14,63)$. Avidin-bound proteins were eluted from the beads using $2 \times$ reducing sample buffer containing $\beta$-mercaptoethanol $\left(5\right.$ minutes; $90^{\circ} \mathrm{C}$ ) and separated by SDS-PAGE for Western blot analysis. Whole cell lysates or tissue homogenates were analyzed similarly as input controls.

Histology and immunohistochemistry/immunofluorescence. Left lung lobes were fixed in PFA and paraffin embedded, and 5- $\mu \mathrm{m}$ sections were stained with H\&E, periodic acid-Schiff (PAS), or Masson's trichrome using standardized protocols. Fixed sections were also immunostained for $\alpha$-SMA (Sigma-Aldrich) and detected using Vectastain Alkaline Phosphatase Universal (Vector Laboratories) per the manufacturer's instructions. Staining intensity was scored by 3 independent investigators ( 0 , no reactivity; 1 , minimal staining, 2, moderate staining; 3 , prominent staining) and positive staining areas were quantified using MetaMorph imaging software (Molecular Devices). Lung sections were evaluated for the presence of DUOX1 protein using $\alpha$-DUOX1 (1:200, provided by F. Miot) and Alexa Fluor 555 goat anti-rabbit IgG (Invitrogen, 1:500) and counterstained with DAPI. Phosphorylated EGFR was visualized using a phospho-EGFR (Tyr1068) rabbit mAb (D7A5; Cell Signaling; 1:125) and visualized by HRP-conjugated secondary antibody and the Vectastain Peroxidase ABC Kit (Vector Laboratories), with hematoxylin counterstaining.

Measurement of airway hyperreactivity. Mice were anesthetized, and tracheas were cannulated and connected to a computer-controlled small animal ventilator (flexiVent; SCIREQ). Mice were paralyzed with pancuronium bromide $(0.8 \mu \mathrm{g} / \mathrm{kg}$ i.p.), and depth of anesthesia was monitored continuously with EKG. Following stabilization and assessment of standard lung volume history, mice were exposed to aerosolized PBS with incremental doses of methacholine $(12.5,25$, and $50 \mathrm{mg} / \mathrm{ml})$, and respiratory impedance was determined using the forced oscillation technique for assessment of frequency-independent Newtonian resistance reflecting the conducting airways $\left(R_{\mathrm{n}}\right)$, tissue dampening $(G)$, and tissue stiffness or elastance $(H)$, using the constant-phase model of impedance (64).

ELISAs. Cell culture supernatants or BAL fluids were analyzed for IL-33, IL-5, IL-13, CXCL1/KC, and AREG using DuoSet ELISAs from R\&D Systems. Serum levels of HDM-specific IgG1 were determined by addition of serial dilutions to ELISA plates precoated with $4 \mu \mathrm{g} / \mathrm{ml}$ HDM and detected with biotinylated antibodies against IgG1 or IgE (BD Biosciences) and streptavidin-HRP. MUC5AC ELISA was performed using a kit (Mouse Mucin-5 Subtype AC) from MyBioSource according to the manufacturer's instructions.

Preparation and ex vivo stimulation of lung single-cell suspensions. Lung right lobes were dissociated into single-cell suspensions using enzymatic digestion and a GentleMACS Dissociator (Miltenyi Biotec Inc.) according to the manufacturer's recommendations. Total cell counts were performed using a Advia cell counter, and single-cell suspensions were cultured at $2 \times 10^{6}$ cells $/ \mathrm{ml}$ in RPMI 1640 in the absence or presence of IL-33 $(10 \mathrm{ng} / \mathrm{ml})$ or HDM $(15 \mu \mathrm{g} / \mathrm{ml})$ for 96 hours, after which supernatants were analyzed for IL-5, IL-13, IL-17A, or AREG by ELISA (R\&D Systems).

Analysis of lung ILC2 populations. Lung single-cell suspensions $\left(1 \times 10^{6}\right.$ lung cells $)$ were stained for lineage-negative and ILC2 surface markers using the lineage-negative cocktail (Ly6G [Ly6C], CD45R [B220], Ter119, CD3, and CD11b; Biolegend), NK1.1-Alexa700 (Biolegend), CD11c-Alexa700 (Biolegend), CD90.2-eflour450 (eBioscience), and Ly6A-PE Cy7 (Biolegend). Dead cells were excluded by UV-Blue Live/Dead stain (Life Technologies). Following staining, cells were fixed in DPBS with 1\% paraformaldehyde and analyzed using a BD LSR II FACS equipped to distinguish as many as 7 fluorophores. Flow data were analyzed using FlowJo (Treestar). For flow sorting of ILC2s, mice were first subjected to 3 successive instillations of IL-33 ( $1 \mu \mathrm{g}$; Biolegend) over 5 days, as described previously (32). Lung single-cell suspensions were performed with the same antibodies described above, and $<2 \times 10^{5}$ ILC2s were sorted using the BD FACS Aria high-speed cell sorter and processed for total RNA isolation and RT-qPCR analysis (65). 
RT-PCR. Total cell or tissue RNA was extracted using TRIzol (Invitrogen) and the RNeasy Mini Kit (Qiagen) and reverse transcribed using M-MLV reverse transcriptase and Oligo(dT)12-16 primer. qPCR was performed using SYBR Green PCR Supermix (Bio-Rad) with predesigned primers (Supplemental Table 1) and normalized to GAPDH using the $\Delta \Delta \mathrm{C}_{\mathrm{T}}$ method.

Statistics. Differences between groups were evaluated by either unpaired 2-tailed $t$ test or 2-way ANOVA with Tukey or Bonferroni post-hoc analysis, depending on composition of data sets, using GraphPad Prism version 6.0 (GraphPad). Differences were considered significant when $P<0.05$.

Study approval. All studies with human subjects were reviewed and approved by the Institutional Review Board of the University of Vermont, and all subjects provided written informed consent. All animal procedures were reviewed and approved by the Animal Care and Use Committee of the University of Vermont.

\section{Author contributions}

$\mathrm{AH}, \mathrm{MH}, \mathrm{DEH}, \mathrm{KD}$, and JLA performed and analyzed the experiments; YMWJH, CGI, and MEP assisted with experimental design and analysis of in vivo studies of HDM-induced inflammation and remodeling; LKL was instrumental in performing the lung functional analyses; AED was responsible for recruitment of human subjects and collection of NECs; MG provided the DUOX1-deficient mice; and AVDV was responsible for the conception and overall supervision of the project and wrote the manuscript. All authors contributed collectively to the planning, discussion, and interpretation of experiments and to the writing of the manuscript.

\section{Acknowledgments}

The authors thank Roxana del Rio for her assistance with the flow cytometry analyses, Nirav Daphtary and Minara Aliyeva for their assistance with the analyses of lung mechanics, and Nicole Bishop and Nicole Bouffard for their assistance with Metamorph analysis. This work was supported by NIH grants (R01 HL085646 and ES021476 to AVDV and R01 HL060014 to YMWJH), an Institutional Development Award Centers of Biomedical Research Excellence grant (P30 GM103532 to CGI), postdoctoral fellowship support to DEH (T32 HL076122 and F32 HL129706), and an internal grant from the University of Vermont College of Medicine.

Address correspondence to: Albert van der Vliet, Department of Pathology and Laboratory Medicine, University of Vermont, HSRF Building Room 216, 149 Beaumont Avenue, Burlington, Vermont 05405-0068, USA. Phone: 802.656.8638; E-mail: albert.van-der-vliet@uvm.edu.

1. Fahy JV. Type 2 inflammation in asthma--present in most, absent in many. Nat Rev Immunol. 2015; 15(1):57-65.

2. Wenzel SE. Asthma phenotypes: the evolution from clinical to molecular approaches. Nat Med. 2012;18(5):716-725

3. Holgate ST. The sentinel role of the airway epithelium in asthma pathogenesis. Immunol Rev. 2011;242(1):205-219.

4. Holtzman MJ, Byers DE, Alexander-Brett J, Wang X. The role of airway epithelial cells and innate immune cells in chronic respiratory disease. Nat Rev Immunol. 2014;14(10):686-698.

5. Xiao C, et al. Defective epithelial barrier function in asthma. J Allergy Clin Immunol. 2011;128(3):549-56.e1

6. Polosa R, et al. Expression of c-erbB receptors and ligands in the bronchial epithelium of asthmatic subjects. $J$ Allergy Clin Immunol. 2002;109(1):75-81.

7. Puddicombe SM, et al. Involvement of the epidermal growth factor receptor in epithelial repair in asthma. FASEB J 2000;14(10):1362-1374.

8. Stefanowicz D, et al. Elevated H3K18 acetylation in airway epithelial cells of asthmatic subjects. Respir Res. 2015;16:95.

9. Enomoto Y, et al. Tissue remodeling induced by hypersecreted epidermal growth factor and amphiregulin in the airway after an acute asthma attack. J Allergy Clin Immunol. 2009;124(5):913-20.e1.

10. Hamilton LM, et al. Altered protein tyrosine phosphorylation in asthmatic bronchial epithelium. Eur Respir J. 2005;25(6):978985 .

11. Hamilton LM, et al. The role of the epidermal growth factor receptor in sustaining neutrophil inflammation in severe asthma. Clin Exp Allergy. 2003;33(2):233-240.

12. Vallath S, Hynds RE, Succony L, Janes SM, Giangreco A. Targeting EGFR signalling in chronic lung disease: therapeutic challenges and opportunities. Eur Respir J. 2014;44(2):513-522.

13. Burgel PR, Nadel JA. Epidermal growth factor receptor-mediated innate immune responses and their roles in airway diseases. Eur Respir J. 2008;32(4):1068-1081.

14. Hristova M, et al. Airway epithelial dual oxidase 1 mediates allergen-induced IL-33 secretion and activation of type 2 immune responses. J Allergy Clin Immunol. 2016;137(5):1545-1556.e11.

15. Le Cras TD, et al. Epithelial EGF receptor signaling mediates airway hyperreactivity and remodeling in a mouse model of chronic asthma. Am J Physiol Lung Cell Mol Physiol. 2011;300(3):L414-L421. 
16. Siddiqui S, et al. The modulation of large airway smooth muscle phenotype and effects of epidermal growth factor receptor inhibition in the repeatedly allergen-challenged rat. Am J Physiol Lung Cell Mol Physiol. 2013;304(12):L853-L862.

17. Hur GY, et al. Potential use of an anticancer drug gefinitib, an EGFR inhibitor, on allergic airway inflammation. Exp Mol Med. 2007;39(3):367-375

18. Li T, Perez-Soler R. Skin toxicities associated with epidermal growth factor receptor inhibitors. Target Oncol. 2009;4(2):107-119.

19. Steuer CE, Ramalingam SS. Targeting EGFR in lung cancer: Lessons learned and future perspectives. Mol Aspects Med. 2015;45:67-73

20. Woodruff PG, et al. Safety and efficacy of an inhaled epidermal growth factor receptor inhibitor (BIBW 2948 BS) in chronic obstructive pulmonary disease. Am J Respir Crit Care Med. 2010;181(5):438-445.

21. Gorissen $\mathrm{SH}$, et al. Dual oxidase-1 is required for airway epithelial cell migration and bronchiolar reepithelialization after injury. Am J Respir Cell Mol Biol. 2013;48(3):337-345.

22. Shao MX, Nadel JA. Dual oxidase 1-dependent MUC5AC mucin expression in cultured human airway epithelial cells. Proc Natl Acad Sci U S A. 2005;102(3):767-772.

23. Sham D, Wesley UV, Hristova M, van der Vliet A. ATP-mediated transactivation of the epidermal growth factor receptor in airway epithelial cells involves DUOX1-dependent oxidation of Src and ADAM17. PLoS One. 2013;8(1):e54391.

24. Yoo SK, Freisinger CM, LeBert DC, Huttenlocher A. Early redox, Src family kinase, and calcium signaling integrate wound responses and tissue regeneration in zebrafish. J Cell Biol. 2012;199(2):225-234.

25. Paulsen CE, et al. Peroxide-dependent sulfenylation of the EGFR catalytic site enhances kinase activity. Nat Chem Biol. 2012;8(1):57-64.

26. Cho DY, et al. Expression of dual oxidases and secreted cytokines in chronic rhinosinusitis. Int Forum Allergy Rhinol. 2013;3(5):376-383.

27. Koff JL, Shao MX, Ueki IF, Nadel JA. Multiple TLRs activate EGFR via a signaling cascade to produce innate immune responses in airway epithelium. Am J Physiol Lung Cell Mol Physiol. 2008;294(6):L1068-L1075.

28. Tully JE, et al. Epithelial NF- $\mathrm{kB}$ orchestrates house dust mite-induced airway inflammation, hyperresponsiveness, and fibrotic remodeling. J Immunol. 2013;191(12):5811-5821.

29. Hammad H, Chieppa M, Perros F, Willart MA, Germain RN, Lambrecht BN. House dust mite allergen induces asthma via Toll-like receptor 4 triggering of airway structural cells. Nat Med. 2009;15(4):410-416.

30. Boots AW, Hristova M, Kasahara DI, Haenen GR, Bast A, van der Vliet A. ATP-mediated activation of the NADPH oxidase DUOX1 mediates airway epithelial responses to bacterial stimuli. J Biol Chem. 2009;284(26):17858-17867.

31. Barlow JL, et al. IL-33 is more potent than IL-25 in provoking IL-13-producing nuocytes (type 2 innate lymphoid cells) and airway contraction. J Allergy Clin Immunol. 2013;132(4):933-941.

32. Klein Wolterink RG, et al. Pulmonary innate lymphoid cells are major producers of IL-5 and IL-13 in murine models of allergic asthma. Eur J Immunol. 2012;42(5):1106-1116.

33. Sonnenberg GF, Artis D. Innate lymphoid cells in the initiation, regulation and resolution of inflammation. Nat Med. 2015;21(7):698-708.

34. Allahverdian S, Harada N, Singhera GK, Knight DA, Dorscheid DR. Secretion of IL-13 by airway epithelial cells enhances epithelial repair via HB-EGF. Am J Respir Cell Mol Biol. 2008;38(2):153-160.

35. Wu CA, Peluso JJ, Zhu L, Lingenheld EG, Walker ST, Puddington L. Bronchial epithelial cells produce IL-5: implications for local immune responses in the airways. Cell Immunol. 2010;264(1):32-41.

36. Altenhöfer S, Radermacher KA, Kleikers PW, Wingler K, Schmidt HH. Evolution of NADPH Oxidase Inhibitors: Selectivity and Mechanisms for Target Engagement. Antioxid Redox Signal. 2015;23(5):406-427.

37. Gianni D, et al. A novel and specific NADPH oxidase-1 (Nox1) small-molecule inhibitor blocks the formation of functional invadopodia in human colon cancer cells. ACS Chem Biol. 2010;5(10):981-993.

38. van der Vliet A. NADPH oxidases in lung biology and pathology: host defense enzymes, and more. Free Radic Biol Med. 2008;44(6):938-955

39. De Deken X, Corvilain B, Dumont JE, Miot F. Roles of DUOX-mediated hydrogen peroxide in metabolism, host defense, and signaling. Antioxid Redox Signal. 2014;20(17):2776-2793.

40. Chang S, Linderholm A, Franzi L, Kenyon N, Grasberger H, Harper R. Dual oxidase regulates neutrophil recruitment in allergic airways. Free Radic Biol Med. 2013;65:38-46.

41. Gour N, Wills-Karp M. IL-4 and IL-13 signaling in allergic airway disease. Cytokine. 2015;75(1):68-78.

42. Evans CM, et al. The polymeric mucin Muc5ac is required for allergic airway hyperreactivity. Nat Commun. 2015;6:6281

43. Erle DJ, Sheppard D. The cell biology of asthma. J Cell Biol. 2014;205(5):621-631.

44. Evans CM, Kim K, Tuvim MJ, Dickey BF. Mucus hypersecretion in asthma: causes and effects. Curr Opin Pulm Med. 2009;15(1):4-11.

45. Harper RW, et al. Differential regulation of dual NADPH oxidases/peroxidases, Duox1 and Duox2, by Th1 and Th2 cytokines in respiratory tract epithelium. FEBS Lett. 2005;579(21):4911-4917.

46. Christianson CA, et al. Persistence of asthma requires multiple feedback circuits involving type 2 innate lymphoid cells and IL-33. J Allergy Clin Immunol. 2015;136(1):59-68.e14.

47. Zaiss DM, Gause WC, Osborne LC, Artis D. Emerging functions of amphiregulin in orchestrating immunity, inflammation, and tissue repair. Immunity. 2015;42(2):216-226.

48. Ooi AT, et al. Identification of an interleukin 13-induced epigenetic signature in allergic airway inflammation. Am J Transl Res 2012;4(2):219-228.

49. Nicodemus-Johnson J, et al. Genome-Wide Methylation Study Identifies an IL-13-induced Epigenetic Signature in Asthmatic Airways. Am J Respir Crit Care Med. 2016;193(4):376-385.

50. Manzo ND, Foster WM, Stripp BR. Amphiregulin-dependent mucous cell metaplasia in a model of nonallergic lung injury. Am J Respir Cell Mol Biol. 2012;47(3):349-357.

51. Kwon J, et al. The nonphagocytic NADPH oxidase Duox1 mediates a positive feedback loop during T cell receptor signaling. Sci Signal. 2010;3(133):ra59. 
52. Rada B, Park JJ, Sil P, Geiszt M, Leto TL. NLRP3 inflammasome activation and interleukin-1 $\beta$ release in macrophages require calcium but are independent of calcium-activated NADPH oxidases. Inflamm Res. 2014;63(10):821-830.

53. van der Vliet A, Janssen-Heininger YM. Hydrogen peroxide as a damage signal in tissue injury and inflammation: murderer, mediator, or messenger? J Cell Biochem. 2014;115(3):427-435.

54. Nussbaum JC, et al. Type 2 innate lymphoid cells control eosinophil homeostasis. Nature. 2013;502(7470):245-248

55. Doherty TA. At the bench: understanding group 2 innate lymphoid cells in disease. J Leukoc Biol. 2015;97(3):455-467.

56. Truong TH, Ung PM, Palde PB, Paulsen CE, Schlessinger A, Carroll KS. Molecular basis for redox activation of epidermal growth factor receptor kinase. Cell Chem Biol. 2016;23(7):837-848.

57. Hoffman SM, et al. Ablation of glutaredoxin-1 modulates house dust mite-induced allergic airways disease in mice. Am J Respir Cell Mol Biol. 2016;55(3):377-386.

58. Heppner DE, Hristova M, Dustin CM, Danyal K, Habibovic A, van der Vliet A. The NADPH oxidases DUOX1 and NOX2 Play Distinct Roles in Redox Regulation of Epidermal Growth Factor Receptor Signaling [published online ahead of print September 20, 2016]. J Biol Chem. doi: 10.1074/jbc.M116.749028.

59. Yankaskas JR, et al. Papilloma virus immortalized tracheal epithelial cells retain a well-differentiated phenotype. Am JPhysiol. 1993;264(5 Pt 1):C1219-C1230.

60. Donkó A, et al. Urothelial cells produce hydrogen peroxide through the activation of Duox1. Free Radic Biol Med. 2010;49(12):2040-2048.

61. Muller L, Brighton LE, Carson JL, Fischer WA 2nd, Jaspers I. Culturing of human nasal epithelial cells at the air liquid interface. J Vis Exp. 2013;80(80):e50646. doi:10.3791/50646.

62. De Deken X, et al. Cloning of two human thyroid cDNAs encoding new members of the NADPH oxidase family. $J$ Biol Chem. 2000;275(30):23227-23233.

63. Klomsiri C, et al. Use of dimedone-based chemical probes for sulfenic acid detection evaluation of conditions affecting probe incorporation into redox-sensitive proteins. Meth Enzymol. 2010;473:77-94.

64. Tomioka S, Bates JH, Irvin CG. Airway and tissue mechanics in a murine model of asthma: alveolar capsule vs. forced oscillations. J Appl Physiol. 2002;93(1):263-270.

65. Ather JL, Foley KL, Suratt BT, Boyson JE, Poynter ME. Airway epithelial NF-kB activation promotes the ability to overcome inhalational antigen tolerance. Clin Exp Allergy. 2015;45(7):1245-1258. 